TRANSACTIONS OF THE

AMERICAN MATHEMATICAL SOCIETY

Volume 360, Number 10, October 2008, Pages 5367-5396

S 0002-9947(08)04454-1

Article electronically published on April 17, 2008

\title{
RELATIVE BEILINSON MONAD AND DIRECT IMAGE FOR FAMILIES OF COHERENT SHEAVES
}

\author{
DAVID EISENBUD AND FRANK-OLAF SCHREYER
}

\begin{abstract}
The higher direct image complex of a coherent sheaf (or finite complex of coherent sheaves) under a projective morphism is a fundamental construction that can be defined via a Cech complex or an injective resolution, both inherently infinite constructions. Using free resolutions it can be defined in finite terms. Using exterior algebras and relative versions of theorems of Beilinson and Bernstein-Gel'fand-Gel'fand, we give an alternate and generally more efficient description in finite terms.

Using this exterior algebra description we can characterize the generic finite free complex of a given shape as the direct image of an easily-described vector bundle. We can also give explicit descriptions of the loci in the base spaces of flat families of sheaves in which some cohomological conditions are satisfied: for example, the loci where vector bundles on projective space split in a certain way, or the loci where a projective morphism has higher dimensional fibers.

Our approach is so explicit that it yields an algorithm suited for computer algebra systems.
\end{abstract}

\section{INTRODUCTION}

Let $A$ be a Noetherian ring, let $\mathcal{F}$ be a coherent sheaf on $\mathbb{P}_{A}^{n}=\mathbb{P}^{n} \times \operatorname{Spec} A$, and let $\pi$ be the projection map $\mathbb{P}_{A}^{n} \rightarrow \operatorname{Spec} A$ :

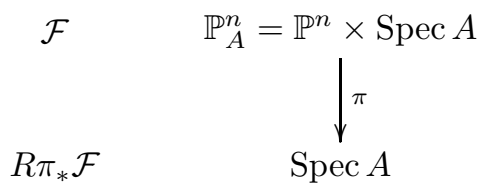

A locally free complex $R \pi_{*} \mathcal{F}$ of coherent sheaves on $\operatorname{Spec} A$ (or, equivalently, of $A$-modules) is defined up to quasi-isomorphism by pushing forward an injective resolution of $\mathcal{F}$ and choosing a bounded complex of finitely generated $A$-modules quasi-isomorphic to it. There is also a description in finite terms using free resolutions over the polynomial ring $A\left[x_{0}, \ldots, x_{n}\right]$ (see Proposition 0.3 ). In this paper we will give a more efficient construction, using (finite parts of) resolutions over an exterior algebra. This makes it possible to compute, for example, the loci in flat families of sheaves where certain cohomological conditions are satisfied, such as the loci where certain decompositions occur in a family of vector bundles, or to detect higher dimensional fibers.

For example, if $\mathcal{F}$ is flat over $A$, and $A$ is local with maximal ideal $\mathfrak{m}$, then by a theorem of Grothendieck Gro63, see also Mumford [Mum70, II.5] or Hartshorne

Received by the editors July 31, 2005 and, in revised form, September 28, 2006.

2000 Mathematics Subject Classification. Primary 14F05, 13 D02. 
Har77, III.12], the complex $R \pi_{*} \mathcal{F} \in D^{b}(A)$ is represented by a minimal complex

$$
0 \rightarrow A^{h^{0}} \rightarrow A^{h^{1}} \rightarrow \ldots \rightarrow A^{h^{n}} \rightarrow 0
$$

of free $A$-modules, which is unique up to isomorphism. Because $\mathcal{F}$ is flat the formation of this complex commutes with base change, and hence

$$
h^{i}=\operatorname{dim}_{K} \mathrm{H}^{i}(\mathcal{F} \otimes K),
$$

where $K=A / \mathfrak{m}$ denotes the residue class field of $A$. If $\mathcal{F}$ is given explicitly, then our techniques algorithmically compute the matrices in this complex.

To formulate the main result we introduce some notation. Let $W=\pi_{*} \mathcal{O}_{\mathbb{P}_{A}^{n}}(1)$ be the free $A$-module of rank $n+1$ underlying $\mathbb{P}_{A}^{n}$, and let $x_{0}, \ldots, x_{n}$ be a basis of $W$. The scheme $\mathbb{P}_{A}^{n}$ is Proj $S$ where $S=\operatorname{Sym} W \cong A\left[x_{0}, \ldots, x_{n}\right]$. Let $M=\sum_{d} M_{d}$ be a graded $S$-module whose sheafification is $\mathcal{F}$, and let $\operatorname{reg}(M)$ denote its CastelnuovoMumford regularity (as defined, in this relative case, below). Let

Let $V=\operatorname{Hom}_{A}(W, A)$ be the dual of $W$, with basis $e_{0}, \ldots, e_{n}$ dual to $x_{0}, \ldots, x_{n}$.

$$
E=\Lambda V=\bigoplus_{i=0}^{n+1} \Lambda^{i} V
$$

be the exterior algebra on $V$. We give the variables $e_{i}$ degree -1 . We write $(V)$ for the augmentation ideal generated by $V$ in $E$.

Any projective $E$-module $M$ can be written in the form $E \otimes_{A} N$, where $N$ is the projective $A$-module $M /(V) M$. More generally, if $N$ is any $A$-module, we say that $E \otimes N$ is a relatively projective $E$-module. We make a similar definition for modules over $S$. There is a well-developed theory of relative homological algebra, including relative projective resolutions, due to Hochschild Hoch56]. We review some elementary facts about it in Section 1. For us relative projectives will be useful because modules generally have smaller resolutions in terms of relative projectives than in terms of projectives.

Corresponding to the graded $S$-module $M$ there is a complex of $E$-modules

$$
\cdots \rightarrow E \otimes M_{d} \rightarrow E \otimes M_{d+1} \rightarrow E \otimes M_{d+2} \rightarrow \cdots
$$

where $\otimes$ denotes $\otimes_{A}$, the module $E \otimes M_{d}$ is in cohomological degree $d$ and is generated by $M_{d}$ regarded as an $A$-module concentrated in degree $d$. The differentials in the complex are given by

$$
a \otimes m \mapsto \sum_{i} e_{i} a \otimes x_{i} m .
$$

In what follows we will write $\sim$ for the quasi-isomorphism of (bounded-above) complexes, i.e., the equivalence relation generated by declaring two complexes quasiisomorphic if there is a map between them that is an isomorphism on homology (for free bounded-above complexes this is the same as homotopy equivalence).

Theorem 0.1 (Main Theorem). Suppose $s \geq \max (0, \operatorname{reg}(M))$, and set $P^{s}=$ $\operatorname{ker}\left(E \otimes M_{s+1} \rightarrow E \otimes M_{s+2}\right)$. If $\mathbf{T}$ is a graded relatively projective resolution of $P^{s}$, regarded as an $E$-module concentrated in cohomological degree $s$, then

$$
R \pi_{*} \mathcal{F} \sim\left(\mathbf{T} \otimes_{E} A\right)_{0} .
$$

Corollary 0.2. With hypotheses and notation as in Theorem 0.1 ,

$$
R^{i} \pi_{*} \mathcal{F} \cong \operatorname{Tor}_{s-i}^{E}\left(P^{s}, A\right)_{0} .
$$


Proof. Take $\mathbf{T}$ to be a projective resolution.

It is interesting to compare the result of Theorem 0.1 with the following more elementary construction of $R \pi_{*} \mathcal{F}$. Here we use a different notion of regularity, derived from a minimal relatively projective $A$-split resolution (see Section 1). It seems reasonable to conjecture that this bound can be improved along the lines of Smith [Sm00], who used the same idea to compute global Ext, and thus the individual $R^{i} \pi_{*}$ functors. However we cannot simply take $d>\operatorname{reg} M$ since this would allow us to take $I=S$ whenever $\operatorname{reg} M \leq n$, leading to a false result.

Theorem 0.3. Assume for simplicity that $n \geq 1$. Let $M$ be a finitely generated $A\left[x_{0}, \ldots, x_{n}\right]$-module, and let $\mathcal{F}$ be the corresponding sheaf. Let $d$ be such that $M$ admits a relatively projective $A$-split resolution by modules $S \otimes N_{i}$ where each $N_{i}$ is a graded $A$-module that is zero in degrees $>d$ (see Theorem 1.2). Suppose also that $I \subset S$ is an ideal such that $S / I$ is projective as an $A$-module, $I$ is contained in $\left(x_{0}, \ldots, x_{n}\right)^{d-n}$, and I contains some power of each $x_{i}$. If $\mathbb{F}$ is a free resolution of $I$, then

$$
R \pi_{*} \mathcal{F} \sim\left(\operatorname{Hom}_{S}(\mathbb{F}, M)\right)_{0} .
$$

Among the examples of ideals $I$ such that $S / I$ is $A$-projective are all ideals generated by monomials and any ideal generated by a homogeneous complete intersection of length $n+1$ that contains a power of each of the $x_{i}$.

We give a proof in Section 1 .

In the first section, we recall some facts about relative projectives and use them to prove Theorem 0.3 .

The next three sections of the paper contain a description of $\mathbf{T}$ and a proof of Theorem 0.1 . Its main ingredients are:

(1) An effective construction of a relative Beilinson monad.

(2) The fact that the relative Beilinson monad for $\mathcal{F}$ is $\pi_{*}$-acyclic; that is, $R^{i} \pi_{*}$ vanishes on all terms of the monads for all $i>0$.

In the remainder of the paper we carry out this construction of the direct image complex in three examples. The first concerns the versal deformation of a rank $r$ vector bundle of the form $\mathcal{O}^{r-1} \oplus \mathcal{O}(d)$ on $\mathbb{P}^{1}$ (this example can, of course, be treated by other means, and we sketch a more elementary alternative). The result gives determinantal equations for the loci, in the base space of the deformation, of bundles of a given splitting type; we conjecture that these determinantal equations actually generate the prime ideals corresponding to the loci in question. The case $r=2$ has a considerable history, and can already be found, in equivalent form and without proof, in Room Roo38.

Our second example treats the direct images of certain sheaves on the resolution of an elliptic singularity; this example seems amenable only to computation.

The last example was the most surprising to us. It leads to a new description of the variety of complexes, in Theorem [5.10, and answers the question, "Which complexes appear as direct images of vector bundles?"

Theorem 0.4. Let $A$ be a Noetherian ring, and let

$$
\mathbb{F}: \quad 0 \rightarrow A^{\beta_{0}} \rightarrow A^{\beta_{1}} \rightarrow \ldots \rightarrow A^{\beta_{n}} \rightarrow 0
$$

be any finite complex of finitely generated free A-modules of length $n$. There is a vector bundle $\mathcal{F}$ on $\mathbb{P}_{A}^{n}$ such that $\mathbb{F}$ represents $R \pi_{*} \mathcal{F}$. 
In fact the bundle $\mathcal{F}$ can be given quite explicitly; see the proof of Theorem 5.9 ,

An implementation of the resulting algorithms in the system Macaulay2 and all the examples treated as illustrations of the main result can be downloaded from http://www.math.uni-sb.de/ag/schreyer/computeralgebra.

Notation. Throughout this paper, $A$ will denote a Noetherian ring and $S$ will denote the polynomial ring $A\left[x_{0}, \ldots, x_{n}\right]=\operatorname{Sym}(W)$, where $W$ is the free $A$-module of rank $n+1$ generated by the $x_{i}$. We grade $S$ with $A$ in degree 0 and the $x_{i}$ in degree 1. There is a canonical projection $\pi: \mathbb{P}_{A}^{n} \rightarrow \operatorname{Spec} A$ corresponding to the inclusion $A \subset S$. We denote by $M$ a finitely generated graded $S$-module, and by $\mathcal{F}=\widetilde{M}$ the associated sheaf on $\mathbb{P}_{A}^{n}=\operatorname{Proj} S$. We write $R \pi_{*} \mathcal{F}$ for the (total) direct image, which is a complex of $A$-modules determined up to quasi-isomorphism.

We write $V$ for the $A$-dual of $W$; the module $V$ is thus a free $A$-module concentrated in degree -1 . We let $e_{0}, \ldots, e_{n}$ denote a basis of $V$ dual to $x_{0}, \ldots, x_{n}$. Let $E$ be the exterior algebra $E=\bigwedge V=A\left\langle e_{0}, \ldots, e_{n}\right\rangle$. If $A$ is a local ring with maximal ideal $\mathfrak{m}$, then $E$ is a local ring with maximal ideal $\mathfrak{m}_{E}=\mathfrak{m} E+\left(e_{0}, \ldots, e_{n}\right)$.

\section{Relative projectives; Proof of Theorem 0.3}

The definitions and results on relative projectives in this section come from Hochschild [Hoch56; we review them for the convenience of the reader.

Let $A$ be a commutative Noetherian ring, and let $B$ be an $A$-algebra. A $B$ module $P$ is said to be relatively projective (with respect to $A$ ) if for every surjective $M \rightarrow M^{\prime}$ of $B$-modules which splits as a map of $A$-modules, the induced map $\operatorname{Hom}_{B}(P, M) \rightarrow \operatorname{Hom}_{B}\left(P, M^{\prime}\right)$ is also surjective; that is, $B$-linear maps from $P$ can be lifted along $A$-split surjections. Modules of the form $B \otimes_{A} N$, where $N$ is any $A$-module, are called relatively free.

Proposition 1.1. A B-module $P$ is relatively projective if and only if it is a direct summand of a relatively free $B$-module. If $B$ is Noetherian and positively (or negatively) graded, with degree 0 component $A$, then every finitely generated graded relatively projective $B$-module is relatively free.

Proof. If $P=B \otimes_{A} N$, then $\operatorname{Hom}_{B}(P, M)=\operatorname{Hom}_{A}(N, M)$ and the relative projectivity of $P$ follows from the definition. It is also immediate that a direct summand of a relatively projective module is relatively projective. If $P$ is any $B$-module, then the natural surjection $B \otimes_{A} P \rightarrow P$ is $A$-split by the map sending $p \in P$ to $1 \otimes p \in B \otimes_{A} P$. Thus if $P$ is relatively projective it is a summand of the relatively free module $B \otimes_{A} P$.

Now suppose that $B$ is graded, and $P$ is a graded relatively projective $B$-module. Since the map $B \rightarrow B / B_{+}=A$ is $A$-split, we may write $P / B_{+} P$ as an $A$-submodule of $P$. The induced map of $B$-modules $B \otimes_{A}\left(P / B_{+} P\right) \rightarrow P$ is surjective by Nakayama's Lemma, and is $A$-split. Since $P$ is relatively projective, we can lift the identity map of $P$ and get a splitting $P \rightarrow B \otimes_{A}\left(P / B_{+} P\right)$. The complementary summand goes to zero under the map $B \otimes_{A}\left(P / B_{+} P\right) \rightarrow P$. But this map becomes an isomorphism modulo $B_{+}$. Using Nakayama's Lemma again, we see that the kernel is zero.

We now return to our basic case, where $S$ and $E$ are the exterior and symmetric algebras over $A$ as in the introduction. 
If $T$ is a graded relatively projective $E$-module, then by Proposition 1.1 we can write $T=E \otimes_{A} N$, where $N=\bigoplus_{j} N_{j}$ is a graded $A$-module, with $N_{j}$ concentrated in degree $j$. Here we regard $A$ as concentrated in degree 0 . We think of $N_{j}$ as the module of "generators of $T$ of degree $j$ ". A relatively projective resolution of a module $M$ is defined to be a resolution by relatively projective modules. This terminology differs a little from Hochschild's: he adds the requirement that the resolution be split exact as a sequence of $A$-modules. We will encounter both sorts of resolutions below, so it seems simpler to require $A$-split exactness explicitly when we need it.

Relative projective resolutions are often smaller than projective resolutions. For example, if $A$ is not a regular ring, then some finitely generated modules over the polynomial ring $S=A\left[x_{0}, \ldots, x_{n}\right]$ do not have finite projective resolutions, but they do have finite relatively projective resolutions:

Theorem 1.2 ([och56, Theorem 3]). Every finitely generated (graded) S-module $M$ has a finite (graded) A-split relatively projective resolution, of length at most $n+1$.

We will use Theorem 1.2 together with the following lemma to prove Theorem 0.3 .

Lemma 1.3. If $X$ is an $S$-module and $N$ is an A-module there is a natural transformation $\operatorname{Hom}_{S}(X, S) \otimes_{A} N \rightarrow \operatorname{Hom}_{S}\left(X, S \otimes_{A} N\right)$ that is an isomorphism whenever $X$ is A-projective.

Proof. The map is the composition $\operatorname{Hom}_{S}(X, S) \otimes_{A} N \cong \operatorname{Hom}_{S}(X, S) \otimes_{S}\left(S \otimes_{A} N\right) \cong$ $\operatorname{Hom}_{S}(X, S) \otimes_{S} \operatorname{Hom}_{S}\left(S, S \otimes_{A} N\right) \rightarrow \operatorname{Hom}_{S}\left(X, S \otimes_{A} N\right)$, where the last map is given by composition of homomorphisms. If $N$ is a free $A$-module, the map is trivially an isomorphism. Thus if $\phi: G_{1} \rightarrow G_{0}$ is an $S$-free presentation of $X$, then we get an isomorphism $\operatorname{Hom}_{S}(\phi, S) \otimes_{A} N \rightarrow \operatorname{Hom}_{S}\left(\phi, S \otimes_{A} N\right)$. The kernel of the map $\operatorname{Hom}_{S}\left(\phi, S \otimes_{A} N\right)$ is $\operatorname{Hom}_{S}\left(X, S \otimes_{A} N\right)$. If $X$ is $A$-projective, then $\phi$ is $A$-split, so the kernel of $\operatorname{Hom}_{S}(\phi, S) \otimes_{A} N$ is equal to $\left(\operatorname{ker} \operatorname{Hom}_{S}(\phi, S)\right) \otimes_{A} N=\operatorname{Hom}_{S}(X, S) \otimes_{A} N$ as required.

Proof of Theorem 0.3 . First, suppose that $M=S(-d)$, the free graded module of rank 1 generated in degree $d$, which has regularity $d$. In this case $\mathcal{F}=\mathcal{O}_{\mathbb{P}_{A}^{n}}(-d)$. At most one $R^{i} \pi_{*} \mathcal{O}_{\mathbb{P}_{A}^{n}}(-d)$ is nonzero, so $R \pi_{*} \mathcal{F}$ is quasi-isomorphic to this nonzero module, as a complex concentrated in cohomological degree $i$.

Our hypotheses on $I$ imply that the projective dimension of $I$ is $n$, and that

$$
\mathrm{H}^{i} \operatorname{Hom}_{S}(\mathbb{F}, M)= \begin{cases}\operatorname{Hom}_{S}(I, S(-d))=S(-d) & \text { if } i=0 ; \\ \operatorname{Hom}_{A}(S / I, A)(-d+n+1) & \text { if } i=n ; \\ 0 & \text { otherwise. }\end{cases}
$$

Taking the degree 0 part we get a projective complex of $A$-modules with just the desired cohomology, establishing the result in this case.

Next, suppose more generally that $M=S \otimes_{A} N$ is relatively projective. As $A$ is concentrated in degree 0 the module $N$ splits as a direct sum of modules of different degrees, so we may as well assume that $N$ is concentrated in a single degree $d=\operatorname{reg} N=\operatorname{reg} \mathcal{F}$.

We may identify the category of sheaves on $\operatorname{Spec} A$ with the category of graded $A$ modules concentrated in degree 0 . Since $\mathcal{F}=\widetilde{M}$ we have $\mathcal{F}=\left(\pi^{*} N(d)\right) \otimes \mathcal{O}_{\mathbb{P}_{A}^{n}}(-d)$, 
whence

$$
R^{i} \pi_{*} \mathcal{F}=N(d) \otimes R^{i} \pi_{*} \mathcal{O}_{\mathbb{P}_{A}^{n}}(-d) .
$$

As before, $R^{i} \pi_{*} \mathcal{F}$ is nonzero for at most one $i$ (which is either 0 or $n$ ), so $R \pi_{*} \mathcal{F}$ is represented by this $A$-module, as a complex concentrated in cohomological degree 0 or $n$.

On the other hand, $\mathbb{F}$ is a complex of projective $A$-modules, so, by Lemma 1.3 ,

$$
\operatorname{Hom}_{S}(\mathbb{F}, S \otimes N)=\operatorname{Hom}_{S}(\mathbb{F}, S(-d)) \otimes N(d) .
$$

By our hypotheses on $I$ the module $S / I$ is $A$-projective, and $\operatorname{Hom}_{S}(\mathbb{F}, S(-d))$ is a resolution of $\operatorname{Hom}_{A}(A / I, A)(n+1-d)$. This is split as a sequence of $A$-modules, so tensoring with $N(d)$ commutes with taking homology. It follows that the tensor product has the desired cohomology.

Finally, we allow $M$ to be an arbitrary finitely generated graded $S$-module. By Hochschild's Theorem 1.2, $M$ has a finite $A$-split relatively projective resolution. We do induction on the "relative projective dimension" of $M$, that is, the minimal length $m$ of such a resolution. The case $m=0$ is the case where $M$ is relatively projective. By Proposition 1.1, $M$ then has the form $S \otimes_{A} N$, and this is the case we have already treated.

Otherwise, let

$$
0 \rightarrow M^{\prime} \rightarrow S \otimes N \rightarrow M \rightarrow 0
$$

be the beginning of an $A$-split resolution of $M$ by relatively projective modules, so that the relative projective dimension of $M^{\prime}$ is $m-1$ and the short exact sequence is $A$-split. Because each module in $\mathbb{F}$ is relatively projective, and because the previous sequence is $A$-split, it follows that

$$
0 \rightarrow \operatorname{Hom}\left(\mathbb{F}, M^{\prime}\right) \rightarrow \operatorname{Hom}(\mathbb{F},(S \otimes N)) \rightarrow \operatorname{Hom}(\mathbb{F}, M) \rightarrow 0
$$

is an exact sequence of complexes, and thus forms a distinguished triangle in the derived category. By induction, the two complexes on the left are the pushforward complexes of the appropriate sheaves, and it follows that the one on the right is too.

\section{Regularity and BGG}

In this section we generalize Castelnuovo-Mumford regularity to the relative case. Propositions 2.3 and 2.5 have no analogues in the absolute case, and seem to be new properties of flat sheaves.

Relative Castelnuovo-Mumford regularity. If $N$ is a graded $S$-module such that $N_{d}=0$ for $d \gg 0$, then we define the regularity of $N$ by

$$
\operatorname{reg}(N)=\max \left\{d \mid N_{d} \neq 0\right\} \text {. }
$$

For a finitely generated graded $S$-module, on the other hand, we define

$$
\operatorname{reg}(M)=\max _{i}\left(\operatorname{reg} \operatorname{Tor}_{i}^{S}(A, M)-i\right) .
$$

In the absolute case, when $A$ is a field, this is the usual definition (see for example Eisenbud [Eis04]). When $M$ is a finitely generated module concentrated in finitely many degrees, so that both definitions above are applicable, they coincide. This can be proved by re-interpreting regularity in terms of local cohomology, as follows. 


\section{Proposition 2.1.}

$$
\operatorname{reg}(M)=\max _{i}\left(\operatorname{reg} \operatorname{Tor}_{i}^{S}(A, M)-i\right)=\max _{j}\left(\operatorname{reg}_{(x)}^{j} M+j\right)
$$

and for each $i$,

$$
\operatorname{reg} \operatorname{Tor}_{i}^{S}(A, M)-i \geq \operatorname{reg} \mathrm{H}_{(x)}^{n+1-i} M+n+1-i
$$

where $(x)$ denotes the ideal $\left(x_{0}, \ldots, x_{n}\right) \subset S$ and $\mathrm{H}_{(x)}^{j}$ is the local cohomology functor.

Since the proof is similar to that in the classical case (see for example Eisenbud Eis04, Corollary 4.5]), we omit it.

Example 2.2 (Regularity is not semicontinuous). The following example is based on the fact that an ideal can have higher regularity than a complete intersection with generators of the same degrees (see Katzman Ka98, the example following Thm 20]). If we set $i=\left(x_{0}^{3}, x_{1}^{3}, x_{0} x_{2}^{2}+x_{1} x_{3}^{2}\right) \subset S_{0}=K\left[x_{0}, \ldots, x_{3}\right]$ the $S_{0} / i$ has regularity 7 , whereas the ideal generated by a regular sequence of 3 cubics has regularity 6 . Consider the family of ideals, with parameter $t$, given by

$$
I=\left(x_{0}^{3}, x_{1}^{3}, x_{0} x_{2}^{2}+x_{1} x_{3}^{2}+t x_{2}^{3}\right) \subset S=K[t]\left[x_{0}, \ldots, x_{3}\right] .
$$

The generators of $I$ form a regular sequence, and it follows that the regularity of $S / I$ in our sense is 6 , whereas the regularity of the special fiber $S /(I, t)$ is 7 .

In general the regularity can go either up or down on specialization, though the following result shows that in the flat case it can only go down.

Proposition 2.3. In the situation above, suppose that $B$ is an $A$-algebra. If either $M$ or $B$ is $A$-flat, then the regularity of $M \otimes_{A} B$ as a module over $S \otimes_{A} B$ is at most the regularity of $M$ as an $S$-module. If $A$ is local, $B$ is the residue class field of $A$, and $M$ is A-flat, then $\operatorname{reg}_{S \otimes_{A} B} M \otimes_{A} B=\operatorname{reg}_{S} M$.

Proof. Let

$$
\mathbb{F}: \quad \cdots \rightarrow F_{i} \rightarrow \cdots \rightarrow F_{0}
$$

be a graded free resolution of $M$ as an $S$-module. By definition $M$ has regularity $r$ if and only if, for every $d>r$ and every $i \geq 0$, the sequence

$$
\left(F_{i+1} \otimes_{S} A\right)_{d+i} \rightarrow \cdots \rightarrow\left(F_{0} \otimes_{S} A\right)_{d+i} \rightarrow 0,
$$

obtained by taking the degree $d+i$ component of the first $i+1$ steps of $\mathbb{F} \otimes_{S} A$, is exact up to and including the term $\left(F_{i} \otimes_{S} A\right)_{d+i}$. The modules in this sequence are all $A$-free, so if the sequence is exact, then it is split exact as a sequence of $A$-modules. This condition is stable under base change to $B$ : the sequence

$$
\left(F_{i+1} \otimes_{S} B\right)_{d+i} \rightarrow \cdots \rightarrow\left(F_{0} \otimes_{S} B\right)_{d+i} \rightarrow 0
$$

is split exact as a sequence of $B$-modules.

Let $T=S \otimes_{A} B$. If $M$ or $B$ is $A$-flat, then the sequence $\mathbb{F} \otimes_{S} T \cong \mathbb{F} \otimes_{A} B$ is exact, and thus it is a $T$-free resolution of $M \otimes_{S} T \cong M \otimes_{A} B$. The regularity of $M \otimes_{A} B$ as a $T$-module is thus computed from the exactness of the sequences $(*)$, and the desired inequality follows.

Under the hypotheses of the last statement of the proposition the other inequality follows from Nakayama's Lemma. 
Bernstein-Gel'fand-Gel'fand correspondence. Using this notion of regularity, we easily generalize the BGG correspondence. We need it in the form given for the case where $A$ is a field, which we studied in EFS03 following BGG78. The proofs are essentially the same as in the case treated there. For the reader's convenience we formulate the necessary statements, which involve a pair of adjoint functors

$$
\operatorname{cplx}(S) \rightleftarrows_{L}^{R} \operatorname{cplx}(E) .
$$

To the graded $S$-module $M$ we associate

$$
\mathbf{R}(M): \quad \cdots \rightarrow \operatorname{Hom}_{A}\left(E, M_{d}\right) \rightarrow \operatorname{Hom}_{A}\left(E, M_{d+1}\right) \rightarrow \cdots,
$$

which is (in all interesting cases) an infinite complex of $E$-modules with the differential given by the action of $t=\sum_{j=0}^{n} x_{j} \otimes e_{j}$. Since

$$
\operatorname{Hom}_{A}(E,-) \cong E \otimes \wedge^{n+1} W \otimes-
$$

canonically, this complex is isomorphic up to a shift in the grading to the complex

$$
\cdots \rightarrow E \otimes M_{d} \rightarrow E \otimes M_{d+1} \rightarrow \cdots
$$

of the introduction. The definition extends to the case where $M$ is a bounded complex of $S$-modules by taking $\mathbf{R}(M)$ to be the total complex of the induced double complex.

Similarly $\mathbf{L}$ is defined by associating with a graded $E$-module $P=\bigoplus_{j} P_{j}$ the complex

$$
\mathbf{L}(P): \quad \cdots \rightarrow S \otimes_{A} P_{j} \rightarrow S \otimes_{A} P_{j-1} \rightarrow \cdots
$$

with $P_{j}$ concentrated in degree $j$, and $S \otimes_{A} P_{j}$ the term of homological degree $j$. For example the complex $\mathbf{L}(E)$ has the form

$$
0 \rightarrow S \rightarrow S^{n+1}(1) \rightarrow \bigwedge^{2}\left(S^{n+1}(1)\right) \rightarrow \cdots \rightarrow \bigwedge^{n+1}\left(S^{n+1}(1)\right) \cong S(n+1) \rightarrow 0,
$$

which is the Koszul complex.

Theorem 2.4. Let $M$ be a finitely generated $S$-module.

a) The truncated complex $\mathbf{R}\left(M_{\geq s}\right)$ is acyclic for $s \geq \operatorname{reg} M$.

b) Suppose $s \geq \operatorname{reg} M$, and set

$$
P=\operatorname{ker}\left(\operatorname{Hom}_{A}\left(E, M_{s+1}\right) \rightarrow \operatorname{Hom}_{A}\left(E, M_{s+2}\right)\right) .
$$

The complex

$$
\mathbf{L}(P): \ldots \rightarrow S \otimes_{A} P_{s+2} \rightarrow S \otimes_{A} P_{s+1} \rightarrow 0
$$

is acyclic and is a resolution of $M_{>s}$ via the map

$$
S \otimes_{A} P_{s+1}=S \otimes \operatorname{Hom}_{A}\left(\Lambda^{0} V, M_{s+1}\right) \rightarrow M_{>s} \text { where } f \otimes_{A} \varphi \mapsto f \varphi(1) .
$$

We use Proposition 2.3 to deduce a property of flat sheaves and modules:

Proposition 2.5. If the sheafification $\mathcal{F}=\widetilde{M}$ is flat over $\operatorname{Spec} A$, then for $s>$ $\operatorname{reg}(M)$ the module $M_{s}$ is projective as an A-module. Moreover, the complex

$$
E \otimes M_{s-1} \rightarrow E \otimes M_{s} \rightarrow E \otimes M_{s+1}
$$

is split exact at $E \otimes M_{s}$ as a complex of A-modules, and thus

$$
P^{s-1}:=\operatorname{ker}\left(E \otimes M_{s} \rightarrow E \otimes M_{s+1}\right)
$$

is projective as an A-module. 
We will use the following well-known result, whose proof we provide for the reader's convenience:

Lemma 2.6. Suppose that $A$ is a commutative Noetherian ring, and

$$
F: 0 \rightarrow M^{\prime} \rightarrow M \rightarrow M^{\prime \prime} \rightarrow 0
$$

is an exact sequence of finitely generated $A$-modules, with $M$ projective. If $F \otimes_{A} A / P$ is exact for all maximal ideals $P$ of $A$, then $F$ is split exact.

It is easy to give examples where the conclusion fails if we do not require $M$ to be projective.

Proof of Lemma 2.6. By the Noetherian and finite generation hypotheses, the formation of $\operatorname{Ext}^{1}\left(M^{\prime \prime}, M^{\prime}\right)$ commutes with localization. Thus it is enough to prove the lemma after localizing $A$ at $P$. We may factor out the largest summand of $M$ contained in $M^{\prime}$, and thus assume that $F$ is the beginning of a minimal free resolution of $M^{\prime \prime}$. In particular, we may assume that $M^{\prime}$ is contained in $P M$. But now tensoring with $A / P$, we see that $M^{\prime} \otimes A / P=0$, so $M^{\prime}=0$ by Nakayama's Lemma.

Proof of Proposition 2.5. From the exactness of the sequence

$$
0 \rightarrow \mathrm{H}_{(x)}^{0} M \rightarrow M \rightarrow \bigoplus_{d \in \mathbb{Z}} \pi_{*} \mathcal{F}(d) \rightarrow \mathrm{H}_{(x)}^{1} M \rightarrow 0
$$

we see that

$$
M_{s} \rightarrow \pi_{*} \mathcal{F}(s)
$$

is an isomorphism for $s>\operatorname{reg}(M)$.

For the first statement of the proposition it thus suffices to show that $\pi_{*} \mathcal{F}(d)=$ $M_{d}$ is flat, given that $\mathcal{G}:=\mathcal{F}(d)$ is flat. This holds very generally: If $I \subset A$ is any ideal, then by the flatness of $\mathcal{G}$ the map $\pi^{-1} I \otimes_{\pi^{-1} A} \mathcal{G} \rightarrow \pi^{-1} A \otimes_{\pi^{-1} A} \mathcal{G}=\mathcal{G}$ is a monomorphism, where we have written $A$ instead of $\mathcal{O}_{\operatorname{Spec} A}$. Writing $\mathcal{O}$ for $\mathcal{O}_{\mathbb{P}_{A}^{n}}$ we see that the composite map

$$
\pi^{*} I \otimes \mathcal{G}=\pi^{-1} I \otimes_{\pi^{-1} A} \mathcal{O} \otimes_{\mathcal{O}} \mathcal{G}=\pi^{-1} I \otimes_{\pi^{-1} A} \mathcal{G} \rightarrow \pi^{-1} A \otimes_{\pi^{-1} A} \mathcal{G}=\mathcal{G}
$$

is a monomorphism. Since the map $I \otimes_{A} \pi_{*} \mathcal{G} \rightarrow \pi_{*} \mathcal{G}$ is the direct image of $\pi^{*} I \otimes \mathcal{G} \rightarrow$ $\mathcal{G}$, it too is a monomorphism, so $\pi_{*} \mathcal{G}$ is flat.

We next must show the sequence

$$
E \otimes M_{s-1} \rightarrow E \otimes M_{s} \rightarrow E \otimes M_{s+1}
$$

is split exact in the middle (that is, the kernel of the right-hand map is equal to the image of the left-hand map, and is a direct summand of $E \otimes M_{s}$.

Exactness is simply the statement of Theorem 2.4 a). By Proposition 2.3, the regularity of $M \otimes A / P$ is also $<s$, so the sequence

$$
E \otimes M_{s-1} \otimes A / P \rightarrow E \otimes M_{s} \otimes A / P \rightarrow E \otimes M_{s+1} \otimes A / P
$$

is also exact. By the first part of the proposition, $M_{s}$ (and thus also $E \otimes M_{s}$ ) is $A$-projective. Lemma 2.6 now completes the proof. 


\section{The Tate Resolution}

We next define and construct Tate resolutions of a sheaf $\mathcal{F}$ on $\mathbb{P}_{A}^{n}$. When $A$ is a field, the Tate resolution is defined in EFS03. by joining the complex $\mathbf{R}\left(M_{>s}\right)$, for $s>\operatorname{reg} M$, to a free resolution of $\operatorname{ker}\left(\operatorname{Hom}_{A}\left(E, M_{s+1}\right) \rightarrow \operatorname{Hom}_{A}\left(E, M_{s+2}\right)\right)$ to make a doubly infinite acyclic complex of free modules, but in the relative case, when $\mathcal{F}$ is not flat, the modules in $\mathbf{R}\left(M_{>s}\right)$ are not free, and the situation is somewhat more subtle. In the general case, the Tate resolution will be a doubly infinite acyclic complex of graded relatively projective $E$-modules.

If $T=\bigoplus_{j} E \otimes_{A} N_{j}$ is a graded, relatively projective $E$-module, with $N_{j}$ concentrated in degree $j$, then we write

$$
T_{\preceq s}:=T /\left(\bigoplus_{j>s} E \otimes_{A} N_{j}\right) \cong \bigoplus_{j \leq s} E \otimes_{A} N_{j}
$$

with $N_{j}$ concentrated in degree $j$, and call it the $s$-th generator truncated quotient of $T$. This notion extends to complexes: if

$$
\mathbf{T}: \quad \cdots \rightarrow T^{i-1} \rightarrow T^{i} \rightarrow \cdots
$$

is a complex of graded $E$-modules of the form $E \otimes_{A} N$ as above, we denote by $\mathbf{T} \preceq s$ the complex formed from the $T_{\prec s}^{i}$. It is a quotient complex of $\mathbf{T}$.

A visual idea of $\mathbf{T}_{\preceq s}$ may help the reader through what follows. We exhibit a sort of Betti diagram for $\mathbf{T}$, in the sense of Bayer and Stillman (see for example Macaulay2), by putting all the $N_{j}^{i}$ in a grid with each column corresponding to a particular $T^{i}$ :

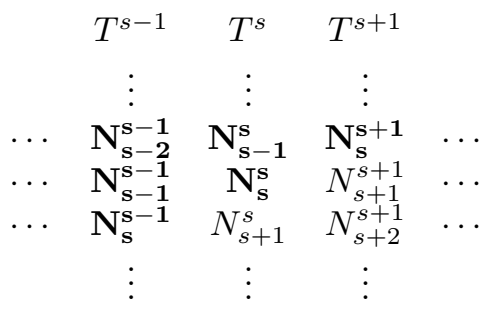

Here the maps go from left to right. Those represented by matrices of scalars (degree 0 in $E$ ) go diagonally up and to the right, $N_{j}^{i} \rightarrow N_{j}^{i+1}$; the maps represented by matrices of linear forms in the variables of $E$ (degree -1$)$ are horizontal, $N_{j}^{i} \rightarrow$ $V \otimes N_{j+1}^{i+1} \subset E \otimes N_{j+1}^{i+1}$, etc. We have indicated the part of the complex belonging to $\mathbf{T}_{\preceq s}$ by putting it in boldface.

As usual, we work with a finitely generated graded $S$-module $M$ and the coherent sheaf $\mathcal{F}=\widetilde{M}$ on $\mathbb{P}_{A}^{n}$ associated with it. We set

$$
P^{s}=\operatorname{ker}\left(E \otimes M_{s+1} \rightarrow E \otimes M_{s+2}\right) .
$$

Note that $P^{s}$ differs from the module $\operatorname{ker}\left(\operatorname{Hom}_{A}\left(E, M_{s+1}\right) \rightarrow \operatorname{Hom}_{A}\left(E, M_{s+2}\right)\right)$ used in the last section only by a shift of degrees, since $\operatorname{Hom}_{A}\left(E, M_{d}\right) \cong \operatorname{Hom}_{A}(E, A)$ $\otimes M_{d} \cong E \otimes \wedge^{n+1} W \otimes M_{d}$, and $\wedge^{n+1} W \cong A(n+1)$.

We define a Tate resolution of $\mathcal{F}$ to be an acyclic, doubly infinite complex $\mathbf{T}=\mathbf{T}(\mathcal{F})$ of graded relatively projective $E$-modules $T^{i}=E \otimes_{A} N^{i}$ such that $\mathbf{T}_{\preceq s}$ is a resolution of $P^{s}$ when $s \gg 0$. A projective Tate resolution is a Tate resolution in which all the $T^{i}$ are projective as $E$-modules or, equivalently, all the $N^{i}$ are projective as $A$-modules. Here $P^{s}$ is concentrated in degree $s+1$, and in cohomological degree $s$; thus we require $\mathbf{T}_{\preceq s}$ to be concentrated in homological 
degrees $\leq s$. In our construction, we will produce a Tate resolution such that $\mathbf{T}_{\preceq s}$ is a resolution of $P^{s}$ for all $s \geq \operatorname{reg} M$ and all the $N^{i}$ are finitely generated. We will also produce a projective Tate resolution with the first property, but it may happen that there are no projective Tate resolutions where all $N^{i}$ are finitely generated.

Construction of Tate resolutions. Let $M$ be any finitely generated graded $S$ module such that $\widetilde{M}=\mathcal{F}$. Choose an integer $s \geq \operatorname{reg} M$, and let

$$
P^{s}=\operatorname{ker}\left(E \otimes M_{s} \rightarrow E \otimes M_{s+1}\right)
$$

as above. Choose a relatively projective resolution

$$
\cdots \rightarrow T^{s-1} \rightarrow T^{s} \rightarrow P^{s} \rightarrow 0 .
$$

Let $\mathbf{T}$ be the complex

$$
\mathbf{T}: \quad \cdots \rightarrow T^{s-1} \rightarrow T^{s} \rightarrow E \otimes_{A} M_{s} \rightarrow E \otimes_{A} M_{s+1} \rightarrow \cdots .
$$

Corollary 3.1. The complex $\mathbf{T}$ above is a Tate resolution for $\mathcal{F}$. If $\mathcal{F}$ is flat over $A$ and the $T^{i}$ are chosen to be projective over $S$, then the complex $\mathbf{T}$ is a projective Tate resolution that is $A$-split.

Proof. Since $s \geq \operatorname{reg} M$, Theorem 2.4 shows that the right-hand part of this complex is acyclic, and the left-hand part is acyclic by construction. For $u \geq s$ the truncations $\mathbf{T}_{\preceq u}$ are resolutions of the desired form. If $\mathcal{F}$ is flat, then the right-hand part

$$
\mathbf{R}\left(M_{\geq s}\right): E \otimes_{A} M_{s} \rightarrow E \otimes_{A} M_{s+1} \rightarrow \cdots
$$

is an $A$-split exact projective complex by Proposition 2.5. In particular $P^{s}$ is $A$ projective, so the left-hand part

$$
\cdots \rightarrow T^{s-1} \rightarrow T^{s}
$$

is also $A$-split.

When $\mathcal{F}$ is not flat over $A$ we will construct a projective Tate resolution by choosing a projective resolution $\mathbf{T}$ of $P^{s}$, for some $s \geq \operatorname{reg} M$, and then extending it inductively to a Tate resolution $\mathbf{T}(\mathcal{F})$ by adding generators in degrees higher than $s$ so that $\mathbf{T}=\mathbf{T}(\mathcal{F})_{\preceq s}$.

Proposition 3.2. Suppose that $s \geq \operatorname{reg} M$ and let $\mathbf{T}$ be a projective resolution of $P^{s}$. Let $F: \ldots \rightarrow F^{s-1} \rightarrow F^{s} \rightarrow F^{s+1} \rightarrow M_{s+1}(s+1) \rightarrow 0$ be a projective resolution as of $M_{s+1}(s+1)$ as an $A$-module. The mapping cone $\mathbf{T}^{\prime}$ of

$$
\mathbf{T} \rightarrow E \otimes_{A} F(-s-1)
$$

is a projective resolution of $P^{s+1}$, and $\mathbf{T}=\mathbf{T}_{\preceq s}^{\prime}$. For $j \leq s$ the inclusion $\mathbf{T} \subset \mathbf{T}^{\prime}$ induces an equality $\left(A \otimes_{E} \mathbf{T}^{\prime}\right)_{j}=\left(A \otimes_{E} \mathbf{T}\right)_{j}$.

If $A$ is local and both $\mathbf{T}$ and $F$ are minimal free resolutions, then so is $\mathbf{T}^{\prime}$.

Proof. By Theorem 2.4 the sequence

$$
0 \rightarrow P^{s} \rightarrow E \otimes M_{s+1} \rightarrow P^{s+1} \rightarrow 0
$$

is exact. The complex $E \otimes F(-s-1)$ is a resolution of the $E$-module $\rightarrow E \otimes M_{s+1}$. Thus the given mapping cone is a projective resolution of $P^{s+1}$ and differs from $\mathbf{T}^{\prime}$ by the addition of free modules generated in degree exactly $s+1$.

In the local case, if $\mathbf{T}^{\prime}$ and $F$ are minimal, then the free modules in $\mathbf{T}^{\prime}$ are all generated in degrees $\leq s$, the generator degree of $P^{s}$, since the elements of $E$ have 
degree $\leq 0$. Thus the comparison maps from $\mathbf{T}(\mathcal{F}) \preceq s$ to $E \otimes F$ cannot contain degree 0 components, and the mapping cone is again minimal.

We can now complete the construction of projective Tate resolutions in general. Let $M$ be a finitely generated graded $S$-module, and let $s>r:=\operatorname{reg} M$ be an integer. Let $\mathcal{F}=\widetilde{M}$ be the corresponding coherent sheaf on $\mathbb{P}_{A}^{n}$. Set $P^{s}=\operatorname{ker} E \otimes$ $M_{s+1} \rightarrow E \otimes M_{s+2}$ as above, and suppose that for $s \geq r$ the $A$-module $M_{s}$ has projective dimension at most $d$ (we allow the case $d=\infty$ ). Let $\mathbf{T}^{r}$ be a projective resolution of $P^{r}$. Supposing that $\mathbf{T}^{s}$ has been constructed for $s \geq r$, let $\mathbf{T}^{s+1}$ be the result of extending $\mathbf{T}^{s}$, as in Proposition 3.2 using a projective resolution of length at most $d$. The union $\mathbf{T}=\mathbf{T}(\mathcal{F})$ of the $\mathbf{T}^{s}$ is a Tate resolution of $\mathcal{F}$.

If $d<\infty$, then each $T^{u}$ will be finitely generated, but otherwise this may not be the case.

Small Tate resolutions. It is interesting, especially from a computational point of view, to construct Tate resolutions that are as small as possible. For this we need a basic fact about the existence of resolutions of the form

$$
\cdots \rightarrow \bigoplus_{j} E \otimes_{A} N_{j}^{-1} \rightarrow \bigoplus_{j} E \otimes_{A} N_{j}^{0} \rightarrow P \rightarrow 0
$$

Proposition 3.3. Let $P$ be a graded E-module. If $s \leq t$ are integers such that $P_{j}=0$ unless $s \leq j \leq t$, then:

(a) The module $P$ has a (not necessarily projective) resolution of the form (*) such that

$$
N_{j}^{i}=0 \text { unless } i \leq 0 \text { and } s+i \leq j \leq t+i .
$$

(b) If $P$, as an $A$-module, has a projective resolution of length $d$, then $P$, as an E-module, has a projective resolution of the form $(*)$ such that

$$
N_{j}^{i}=0 \text { unless } i \leq 0 \text { and } s+i \leq j \leq \min (t, t+d+i) .
$$

It is easy to give examples where these inequalities are sharp.

Proof. First consider the case $s=t$. The Cartan-Eilenberg resolution of $A$ over $E$,

$$
\mathbf{C E}: \quad \cdots \rightarrow E \otimes\left(\mathrm{Sym}_{2} W\right)^{*} \rightarrow E \otimes W^{*} \rightarrow E \rightarrow A \rightarrow 0
$$

has $\left(\operatorname{Sym}_{m} W\right)^{*}$ concentrated in degree $-m$ for each $m$. The modules in the complex $\mathbf{C E} \otimes_{A} P$ have the form $E \otimes N_{t+i}^{i}$, for $i \leq 0$, where $N_{t+i}^{i}$ is a direct sum of finitely many copies of $P$. We have

$$
\mathrm{H}^{i}\left(\mathbf{C E} \otimes_{A} P\right)=\operatorname{Tor}_{-i}^{E}(A, P)=0 \text { for } i<0,
$$

so $\mathbf{C E} \otimes_{A} P$ is a resolution satisfying part $(a)$.

If

$$
\mathbf{F}: \cdots \rightarrow F^{-1} \rightarrow F^{0} \rightarrow P \rightarrow 0
$$

is a projective resolution of $P=P_{t}$, so that each $F^{i}$ is concentrated in degree $t$, then the homology of the projective complex $\mathrm{H}^{i}\left(\mathbf{C E} \otimes_{A} \mathbf{F}\right)$ is also equal to $\operatorname{Tor}_{-i}^{E}(A, P)=0$, so $\mathbf{C E} \otimes_{A} \mathbf{F}$ is a projective resolution of $A \otimes P$ as an $E$-module. If $F$ has length $d$, the $i$-th term is

$$
T^{i}=\bigoplus_{\substack{u, v \leq 0 \\
u+v=i \\
-d \leq v}} C E^{u} \otimes_{A} F^{v}=\bigoplus_{\begin{array}{c}
u, v \leq 0 \\
u+v=i \\
-d \leq v
\end{array}} E \otimes_{A}\left(\operatorname{Sym}_{-u} W\right)^{*} \otimes_{A} F^{v}
$$


It has generating projective $A$-module

$$
N_{t+j}^{i}=\left(\operatorname{Sym}_{-j} W\right)^{*} \otimes_{A} F^{i-j},
$$

which is nonzero only when $-j \geq 0,-d \leq i-j \leq 0$, that is, in the range $j=$ $t+i, \ldots, \min (t, t+d+i)$, as required for part $(b)$.

For the general case, we do induction on the difference $t-s$, so we may assume, for either statement $(a)$ or $(b)$, that we have complexes $T^{\prime}$ and $T^{\prime \prime}$ of the desired form for the modules $P^{\prime}=P_{s}$ and $P^{\prime \prime}=P / P_{s}$. We can construct from these a resolution $T$ of $P$ whose $i$-th term is $\left(T^{\prime}\right)^{i} \oplus\left(T^{\prime \prime}\right)^{i}$, and this complex will have the desired form.

To construct $T$ in case $(a)$, where the terms of $T^{\prime \prime}$ are not assumed projective, but simply of the form $E \otimes_{A} N$, we must use the fact that, as an $A$-module, $P_{s}$ is a summand of $P$ so that the exact sequence

$$
0 \rightarrow P^{\prime} \rightarrow P \rightarrow P^{\prime \prime} \rightarrow 0
$$

is $A$-split.

We now construct the resolution of $P$ inductively. Let

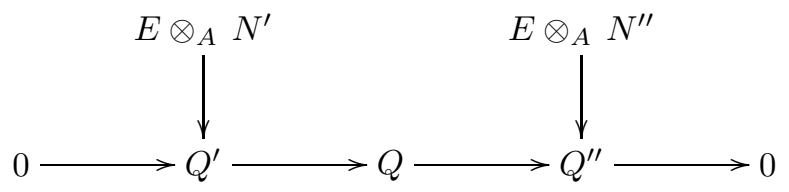

be given with the bottom sequence $A$-split. Because $E \otimes N$ is relatively projective we may lift the map $E \otimes N \rightarrow Q^{\prime \prime}$ to a map $E \otimes N \rightarrow Q$, and use it and the given map on the left to construct a diagram

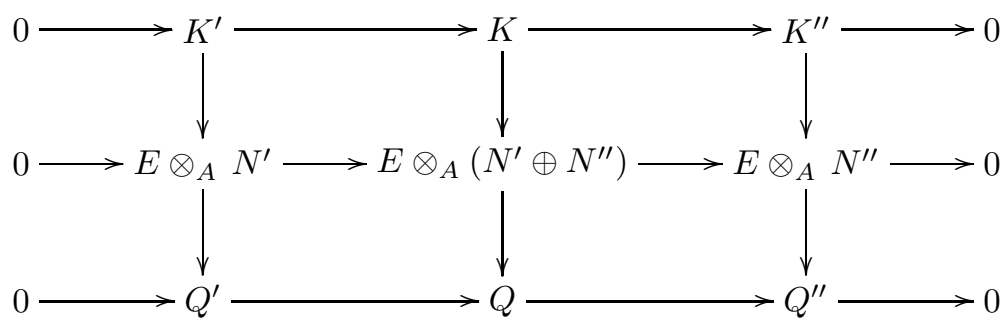

where the modules $K^{\prime}, K, K^{\prime \prime}$ are by definition the kernels of the vertical maps below them. The middle row and the bottom row are compatibly $A$-split, so the exact sequence $0 \rightarrow K^{\prime} \rightarrow K \rightarrow K^{\prime \prime} \rightarrow 0$ is also $A$-split, and we may continue the construction.

The construction of $T$ in the case where the complexes $T^{\prime}$ and $T^{\prime \prime}$ are projective works the same way, and is of course standard.

Corollary 3.4. Let $\mathcal{F}$ be a coherent sheaf on $\mathbb{P}_{A}^{n}$ represented by a finitely generated graded $S$-module $M$ of regularity $r$. The sheaf $\mathcal{F}$ has a Tate resolution $\mathbf{T}$ with terms $T^{i}=\bigoplus_{j} E \otimes_{A} N_{j}^{i}$ such that

$$
N_{j}^{i} \text { is nonzero only in the range } i-n \leq j \leq i,
$$

while if $i>r$, then

$$
N_{j}^{i} \text { is nonzero only if } j=i \text {. }
$$


If every A-module has projective dimension $\leq d \leq \infty$, then there is a Tate resolution of $\mathcal{F}$ such that each $N_{j}^{i}$ is projective, and

$$
N_{j}^{i} \text { is nonzero only in the range } i-n \leq j \leq \min (r, i+d),
$$

while if $i>r$, then

$$
N_{j}^{i} \text { is nonzero only in the range } i \leq j \leq i+d .
$$

In the general case, where $A$ does not have finite global dimension and $\mathcal{F}$ is not flat, the modules $\mathbf{T}(\mathcal{F})^{i}$ in the projective Tate resolution we have constructed will not be finitely generated. However, the module $\left(\mathbf{T}(\mathcal{F})_{\preceq s}\right)^{k}$ is finitely generated. To compute $\left(A \otimes_{E} \mathbf{T}(\mathcal{F})\right)_{u}$, for any $u$, it suffices to compute finitely many terms of the complex $\mathbf{T}^{s}=\mathbf{T}(\mathcal{F})_{\preceq s}$, for example as a resolution of $P^{s}$, for any $s \geq$ $\max (\operatorname{reg} M, u)$.

Proof. The limits follow immediately from our construction, using Proposition 3.3 and Proposition 3.2 .

We can express the restrictions of Corollary 3.4 pictorially in terms of a Betti diagram:

$$
\begin{aligned}
& \begin{array}{llllllll}
\ldots & \ldots & \ldots & \ldots & \ldots & N_{0}^{n} & \ldots & N_{r-1-n}^{r-1}
\end{array} \\
& \begin{array}{lllllllll} 
& \ldots & \ldots & \ldots & \ldots & . & \ldots & \ldots & \vdots
\end{array} \\
& \begin{array}{llllllll}
\cdots & \cdots & \cdots & N_{0}^{1} & \cdots & \cdots & \cdots & N_{r-2}^{r-1}
\end{array} \\
& \begin{array}{llllllllllll}
\cdots & \cdots & N_{0}^{0} & \ldots & \ldots & \ldots & \cdots & N_{r-1}^{r-1} & N_{r}^{r} & \cdots & \cdots & N_{s}^{s} .
\end{array} \\
& \begin{array}{lllllllllll}
\cdots & N_{0}^{-1} & \ldots & \cdots & \cdots & \cdots & \cdots & N_{r}^{r-1} & \cdots & \cdots & N_{s}^{s-1}
\end{array} \\
& \begin{array}{llllllllll}
. & \ldots & \ldots & \ldots & \ldots & \ldots & N_{r}^{r-2} & \ldots & \ldots & .
\end{array} \\
& \begin{array}{lllllllll}
\ldots & \ldots & \ldots & \ldots & \ldots & . & \ldots & \ldots & .
\end{array}
\end{aligned}
$$

In case $A$ is local, the $N_{j}^{i}$ are free, and we sometimes put just the numbers $\operatorname{rank} N_{j}^{i}$ into the table.

The $i$-th column in the table corresponds to the term $\mathbf{T}(\mathcal{F})^{i}$. Maps pointing directly to the right are linear in the variables $e_{i}$, while maps of higher degree in the $e_{i}$ point to the right and downwards. The degree 0 maps in $\mathbf{T}$ go up and to the right, along the $45^{\circ}$ diagonals; thus the complex $\left(A \otimes_{E} \mathbf{T}_{\preceq s}\right)_{k}$ is

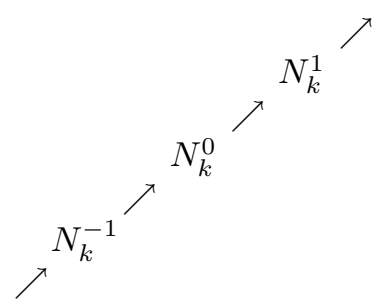

The empty spaces in the table, and in particular the absence of dots above the top row shown, indicate that the terms that might have occurred there are 0 ; these are the restrictions of Corollary 3.4 (in case $d=\infty$ ). 


\section{The Beilinson MONAD}

In this section we use a relatively projective Tate resolution $\mathbf{T}$ for $\mathcal{F}$ to construct a relative Beilinson monad for $\mathcal{F}$ : it is a complex $\mathbf{U}(\mathcal{F})$ of $\pi_{*}$-acyclic coherent sheaves on $\mathbb{P}_{A}^{n}$, whose only homology is $\mathcal{F}$. The construction is parallel to that given in ES03, but involves new subtleties, especially when $\mathcal{F}$ is not flat over $A$.

Let $U$ denote the universal subbundle on $\mathbb{P}_{A}^{n}$ :

$$
0 \rightarrow U \rightarrow W \otimes \mathcal{O} \rightarrow \mathcal{O}(1) \rightarrow 0,
$$

so that $U=\Omega_{\mathbb{P}_{A}^{n} / \operatorname{Spec} A}^{1}(1)$. We will define an additive functor

$$
\mathbf{U}:\{\text { relatively projective } E \text {-modules }\} \rightarrow \operatorname{coh}\left(\mathbb{P}_{A}^{n}\right)
$$

from the category of relatively projective $E$-modules to the category of sheaves on $\mathbb{P}_{A}^{n}$ sending $E \otimes N_{j}$ to $\wedge^{j} U \otimes \pi^{*} N_{j}(j)$. Here we have written $\pi^{*} N_{j}(j)$ instead of $\pi^{*} N_{j}$ because we identify the category of quasi-coherent sheaves on $\operatorname{Spec} A$ with the category of $A$-modules concentrated in degree 0 .

To define the action of $\mathbf{U}$ on morphisms, note that

$$
\operatorname{Hom}_{E}\left(E \otimes N_{j}, E \otimes N_{\ell}\right)=\Lambda^{\ell-j} V \otimes_{A} \operatorname{Hom}_{A}\left(N_{j}(j), N_{\ell}(\ell)\right)
$$

where as always it is understood that $N_{j}$ sits in degree $j$ and $N_{\ell}$ in degree $\ell$. A morphism $\phi \in \Lambda^{\ell-j} V \otimes \operatorname{Hom}_{A}\left(N_{j}(j), N_{\ell}(\ell)\right)$ defines, for each $p$, a morphism $\wedge^{p} W \otimes \pi^{*} N_{j}(j) \rightarrow \wedge^{p+j-\ell} W \otimes \pi^{*} N_{\ell}(\ell)$, and since these morphisms commute with the differentials of the Koszul complex, they induce a map $\mathbf{U}(\phi)$ that makes the following diagram commute (see [ES03, for more details):
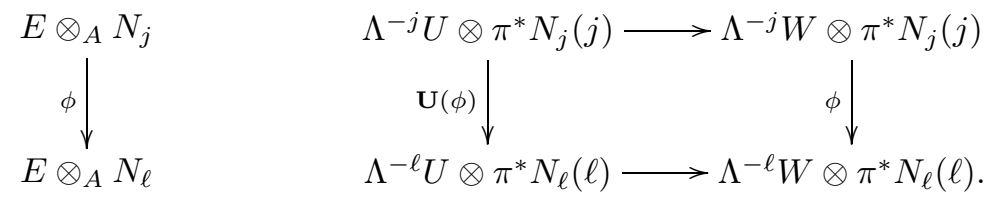

Note that $\Lambda^{-j} U=0$ unless $0 \geq j \geq-n$. Because of this the functor $\mathbf{U}$ will annihilate many terms of a Tate resolution $\mathbf{T}(\mathcal{F})$ so

$$
\mathbf{U}(\mathcal{F}):=\mathbf{U}(\mathbf{T}(\mathcal{F}))
$$

is a bounded-above complex that depends only on $n+1$ diagonals of $\mathbf{T}(\mathcal{F})$. In particular $\mathbf{U}(\mathcal{F})=\mathbf{U}\left(\mathbf{T}(\mathcal{F})_{\preceq s}\right)$ for any $s \geq \max (0, \operatorname{reg}(M))$.

Recall that a monad for $\mathcal{F}$ is a complex $U$ of sheaves whose homology is 0 except for $H^{0}(U)=\mathcal{F}$.

Theorem 4.1. Let $M=\sum_{d} M_{d}$ be a finitely generated graded $S$-module and let $\mathcal{F}$ be the associated coherent sheaf on $\mathbb{P}_{A}^{n}$. If $\mathbf{T}(\mathcal{F})$ is a Tate resolution for $\mathcal{F}$, then the complex $\mathbf{U}=\mathbf{U}(\mathbf{T}(\mathcal{F}))$ is a bounded-above monad for $\mathcal{F}$ whose terms are $\pi_{*}$-acyclic coherent sheaves. There are relatively projective Tate resolutions $\mathbf{T}$ for $\mathcal{F}$ such that $\mathbf{U}$ is a finite complex. On the other hand, if $A$ is local, then $\mathbf{U}$ may be determined by $\mathcal{F}$, uniquely up to isomorphism, by the additional requirement that $\mathbf{T}(\mathcal{F})_{\preceq s}$ is a minimal free resolution for every $s>\operatorname{reg} M$. Furthermore, if

(1) $\mathcal{F}$ is A-flat, or

(2) $\operatorname{pd} A<\infty$

holds, then we can choose a projective Tate resolution such that the complex $\mathbf{U}(\mathcal{F})$ is finite. 
Proof. The sheaf $\wedge^{j} U$ is resolved by a truncated Koszul complex,

$$
0 \rightarrow \Lambda^{n+1} W \otimes \mathcal{O}(i-n-1) \rightarrow \cdots \rightarrow \Lambda^{i+1} W \otimes \mathcal{O}(-1) \rightarrow \Lambda^{i} U \rightarrow 0 .
$$

We deduce, just as in the case where $A$ is a field, that $R^{j} \pi_{*} \Lambda^{i} U=0$ for $i>0$ and all $j$ and that $R \pi_{*} \Lambda^{0} U=R^{0} \pi_{*} \mathcal{O}_{\mathbb{P}_{A}^{n}}=A$. Thus all terms in the complex $\mathbf{U}(\mathcal{F})_{\preceq s}$ are $\pi_{*}$-acyclic.

To prove that $\mathrm{H}^{*}(\mathbf{U}(\mathcal{F}))=\mathrm{H}^{0}(\mathbf{U}(\mathcal{F})) \cong \mathcal{F}$ we use a double complex argument. If $G$ is a graded $S$-module or a complex of such, we let $\widetilde{G}$ stand for the sheafification of a (complex) of graded $S$-modules $G$.

Theorem 2.4 says that $\widetilde{\mathbf{L}}\left(P^{s} \otimes \Lambda^{n+1} W\right)$ is a complex with $\mathcal{F}$ as its only homology. Consider the subcomplex $\left(\mathbf{T}(\mathcal{F})_{\preceq s}\right)_{\geq-n}$ formed by replacing each

$$
\left(T^{i}\right)_{\preceq s}=\bigoplus_{j \leq s} E \otimes_{A} N_{j}^{i}
$$

by the submodule

$$
\left(\left(T^{i}\right)_{s s}\right)_{<-n}=\bigoplus_{j \leq s, \ell \geq n+j} \wedge^{\ell} V \otimes_{A} N_{j}^{i}
$$

of elements in $\mathbf{T}(\mathcal{F})_{\preceq_{s}}$ of degree $\geq-n$. We apply the functor $\widetilde{\mathbf{L}}$ to this subcomplex. The resulting double complex has as vertical complexes $\mathbf{L}\left(\left(\left(T^{i}\right)_{\preceq s}\right)_{<-n}\right)$ that are sums of sheafifications of possibly truncated Koszul complexes $\widetilde{L}\left(\left(E \otimes N_{j}^{i}\right)_{\geq-n}\right)$ as in the following diagram:

$$
\begin{array}{ccc}
0 & 0 \\
\uparrow & \uparrow & \uparrow \\
\sum_{\ell-j=n} \pi^{*}\left(\Lambda^{\ell} V \otimes N_{j}^{i}(j)\right) \otimes \mathcal{O}(n) & \rightarrow & \sum_{\ell-j=n} \pi^{*}\left(\Lambda^{\ell} V \otimes N_{j}^{i+1}(j)\right) \otimes \mathcal{O}(n) \\
\uparrow & & \uparrow \\
\sum_{\ell-j=n-1} \pi^{*}\left(\Lambda^{\ell} V \otimes N_{j}^{i}(j)\right) \otimes \mathcal{O}(n-1) & \rightarrow & \sum_{\ell-j=n-1} \pi^{*}\left(\Lambda^{\ell} V \otimes N_{j}^{i+1}(j)\right) \otimes \mathcal{O}(n-1) \\
\uparrow & & \uparrow
\end{array}
$$

The complex $\widetilde{L}\left(\left(E \otimes N_{j}^{i}\right)_{\geq-n}\right)$ is a resolution of $\Lambda^{-j} U \otimes \pi^{*}\left(N_{j}^{i}(j)\right) \otimes \mathcal{O}(n+1)$ in case $0 \geq j \geq-n$, and otherwise it is exact. Thus the vertical homology of the double complex is the complex $\mathbf{U}(\mathcal{F}) \otimes \mathcal{O}(n+1)$.

On the other hand, since $\mathbf{T}(\mathcal{F})_{\preceq s+1}$ is a resolution, the horizontal homology of $\mathbf{L}\left(\left(\left(T^{i}\right)_{s s}\right)_{<-n}\right)$ is the complex $\widetilde{L}\left(P^{s}\right)$. In the notation of Theorem 2.4, we have $P^{s}=P(-n-1)$, so by that result the homology of $\widetilde{L}\left(P^{s}\right)$ is $\mathcal{F}(n+1)$.

A diagram chase in the double complex proves $\mathrm{H}^{*}(\mathbf{U}(\mathcal{F}) \otimes \mathcal{O}(n+1))=$ $\mathrm{H}^{0}(\mathbf{U}(\mathcal{F}) \otimes \mathcal{O}(n+1)) \cong \mathcal{F}(n+1)$ as desired.

The last statement follows from Corollary 3.4 and Proposition 2.5.

Corollary 4.2. If $\mathbf{T}(\mathcal{F})$ is any Tate resolution for $\mathcal{F}$, then the complex $\pi_{*} \mathbf{U}(\mathbf{T}(\mathcal{F})$ ) represents $R \pi_{*} \mathcal{F}$ in the derived category of bounded-above, finitely generated complexes of $A$-modules. In particular $\pi_{*} \mathbf{U}(\mathcal{F})$ has no homology in negative degrees and $R^{i} \pi_{*} \mathcal{F}=\mathrm{H}^{i} \pi_{*} \mathbf{U}(\mathcal{F})$ for $i \geq 0$. In case $A$ is local and we choose $\mathbf{T}(\mathcal{F})_{\preceq s}$ to be a minimal free resolution, then $\pi_{*} \mathbf{U}(\mathcal{F})$ is the unique minimal free representative. 
Proof. We have exact complexes

$$
\begin{aligned}
\ldots & \rightarrow \mathbf{U}(\mathcal{F})^{-2} \rightarrow \mathbf{U}(\mathcal{F})^{-1} \rightarrow \mathcal{B} \rightarrow 0, \\
0 & \rightarrow \mathcal{K} \rightarrow \mathbf{U}(\mathcal{F})^{0} \rightarrow \mathbf{U}(\mathcal{F})^{1} \rightarrow \ldots
\end{aligned}
$$

and

$$
0 \rightarrow \mathcal{B} \rightarrow \mathcal{K} \rightarrow \mathcal{F} \rightarrow 0 .
$$

Since $\mathbf{U}(\mathcal{F})$ is $\pi_{*}$-acyclic and $R^{n+1} \pi_{*} \mathcal{G}=0$ for any coherent sheaf we get an exact complex $\ldots \rightarrow \pi_{*} \mathbf{U}(\mathcal{F})^{-2} \rightarrow \pi_{*} \mathbf{U}(\mathcal{F})^{-1} \rightarrow \pi_{*} \mathcal{B} \rightarrow 0$ and $R^{i} \pi_{*} \mathcal{B}=0$ for $i>0$. Thus the result follows from

$$
0 \rightarrow \pi_{*} \mathcal{B} \rightarrow R \pi_{*} \mathcal{K} \rightarrow R \pi_{*} \mathcal{F} \rightarrow 0
$$

and $R \pi_{*} \mathcal{K}=\pi_{*} \mathbf{U}(\mathcal{F}) \geq 0$.

Completion of the proof of Theorem 0.1 . Since

we obtain

$$
\pi_{*} \Lambda^{\ell} U= \begin{cases}0 & \text { if } \ell \neq 0 \\ A & \text { if } \ell=0\end{cases}
$$

as desired.

$$
R \pi_{*} \mathcal{F}=\pi_{*} \mathbf{U}(\mathcal{F})=\left(\mathbf{T}(\mathcal{F})_{\preceq_{s}} \otimes_{E} A\right)_{0},
$$

Remark 4.3. Let $\mathcal{B}^{0}=\operatorname{coker}\left(\mathbf{U}(\mathcal{F})^{-1} \rightarrow \mathbf{U}(\mathcal{F})^{0}\right)$. The proof shows that

$$
0 \rightarrow \pi_{*} \mathcal{B}^{0} \rightarrow \pi_{*} \mathbf{U}(\mathcal{F})^{1} \rightarrow \ldots \rightarrow \pi_{*} \mathbf{U}(\mathcal{F})^{n} \rightarrow 0
$$

is a bounded complex representing $R \pi_{*} \mathcal{F}$.

Grothendieck's motivation for introducing $R \pi_{*} \mathcal{F}$ as a complex was to make a base change property true. It is amusing to note that the property follows directly from our construction.

Theorem 4.4 (Base change). Suppose $\mathcal{F}$ is flat over $A$. Then $R \pi_{*} \mathcal{F}$ commutes with base change in the sense that $\varphi^{*} R \pi_{*}(\mathcal{F})$ represents $R \pi_{*}\left(\widetilde{\varphi}^{*} \mathcal{F}\right)$ for any ring homomorphism $\varphi: A \rightarrow A^{\prime}$ in the induced diagram

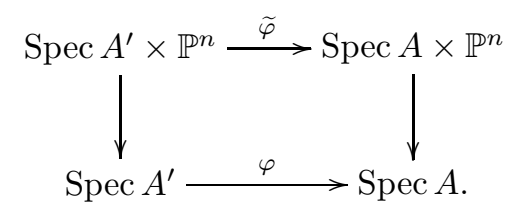

Proof. In the flat case, Corollary 3.1 produces a Tate resolution that commutes with arbitrary base change, since it is split exact as a complex of $A$-modules.

Corollary 4.5. Suppose $A$ is local with maximal ideal $\mathfrak{m}$ and $\mathcal{F}$ is flat over $A$. Then the $k$-th summands in the minimal Tate resolution and Beilinson monad are

$$
(\mathbf{T}(\mathcal{F}))^{k}=\sum_{i=0}^{n}(E \otimes A(-k+i))^{h^{i}(k-i)}
$$

and

$$
(\mathbf{U}(\mathcal{F}))^{k}=\sum_{i=0}^{n}\left(\wedge^{i-k} U\right)^{h^{i}(k-i)},
$$

where $h^{i}(k-i)=\operatorname{dim}_{A / \mathfrak{m}} \mathrm{H}^{i}\left(\mathcal{F}(k-i) \otimes_{A} A / \mathfrak{m}\right)$, as in the case where $A$ is a field. 


\section{EXAmples}

Example 5.1. To exhibit our technique in a simple and natural case, we take the versal deformation $\mathcal{F}$ of the bundle $\mathcal{E}=\mathcal{O} \oplus \mathcal{O}(-2)$, where $\mathcal{O}=\mathcal{O}_{\mathbb{P}^{1}}$ is the structure sheaf of the projective line over a field $K$, and compute the complex $R \pi_{*} \mathcal{F}$.

The base space of this deformation has as tangent space

$$
\operatorname{Ext}^{1}(\mathcal{E}, \mathcal{E}) \cong \mathrm{H}^{1}(\operatorname{Hom}(\mathcal{E}, \mathcal{E}))=\mathrm{H}^{1}(\operatorname{Hom}(\mathcal{O}, \mathcal{O}(-2)))=K,
$$

and since the deformations are unobstructed the base space of the versal deformation is the germ of $\mathbb{A}^{1}=\operatorname{Spec} K[a]$. We thus work over $A=K[[a]]$.

By Corollary 4.5 the Betti diagram of the Tate resolution $\mathbf{T}(\mathcal{F})$ is

\begin{tabular}{r|cccccc}
$i-j \backslash i$ & -2 & -1 & 0 & 1 & 2 & 3 \\
\hline 1 & 6 & 4 & 2 & 1 & & \\
0 & & & 1 & 2 & 4 & 6
\end{tabular}.

Hence the minimal representative of $R \pi_{*} \mathcal{F}$ is the complex

$$
0 \longrightarrow A^{1} \stackrel{\alpha}{\longrightarrow} A^{1} \longrightarrow 0
$$

for some map $\alpha$ (which is not hard to guess, but which we will compute to illustrate our method in this easy example).

From the Betti diagram we see directly that the regularity of $\mathcal{F}$ is 2 , and we can compute $R \pi_{*} \mathcal{F}$ starting from the map of modules

$$
\phi_{2}: E \otimes\left(\mathrm{H}^{0} \mathcal{F}(2)\right) \rightarrow E \otimes\left(\mathrm{H}^{0} \mathcal{F}(3)\right)
$$

over the exterior algebra $E$.

We write $x, y \in W=\pi_{*} \mathcal{O}(1)$ for fiber coordinates on $\mathbb{P}_{A}^{1}$, where now $\mathcal{O}$ denotes the structure sheaf of $\mathbb{P}_{A}^{1}$, and $e, f$ for their dual coordinates in $E$. The sheaf $\mathcal{F}(2)$ is an extension

$$
0 \rightarrow \mathcal{O} \rightarrow \mathcal{F}(2) \rightarrow \mathcal{O}(2) \rightarrow 0 .
$$

Lifting a basis for $\mathrm{H}^{0} \mathcal{O}(2)$, we may choose a basis of the free $A$-module $\mathrm{H}^{0} \mathcal{F}(2)$ denoted by $1, x^{2}, x y, y^{2}$. In terms of this basis, a presentation matrix may be written as

$$
\begin{aligned}
& 1 \\
& x^{2} \\
& x y \\
& y^{2}
\end{aligned}\left(\begin{array}{cc}
-a x & 0 \\
y & 0 \\
-x & y \\
0 & -x
\end{array}\right) .
$$

We choose as basis of $\mathrm{H}^{0} \mathcal{F}(3)$ the elements

$$
x \cdot 1, y \cdot 1, x \cdot x^{2}, x \cdot x y, x \cdot y^{2}, y \cdot y^{2} .
$$

From the given relations we see that $y \cdot x y=x \cdot y^{2}$. However, $y \cdot x^{2}=x \cdot x y+a(x \cdot 1)$. Thus, in terms of these bases, the map $\phi_{2}$ has matrix

$$
\begin{aligned}
& x \cdot 1 \\
& y \cdot 1 \\
& x \cdot x^{2} \\
& x \cdot x y \\
& x \cdot y^{2} \\
& y \cdot y^{2}
\end{aligned} \quad\left(\begin{array}{cccc}
e & a f & 0 & 0 \\
f & 0 & 0 & 0 \\
0 & e & 0 & 0 \\
0 & f & e & 0 \\
0 & 0 & f & e \\
& 0 & f
\end{array}\right) .
$$


The further syzygy matrices are

$$
\phi_{1}=\left(\begin{array}{ccc}
e f & a f & 0 \\
0 & e & 0 \\
0 & f & e \\
0 & 0 & f
\end{array}\right), \quad \phi_{0}=\left(\begin{array}{ccc}
a & e & f \\
e & 0 & 0 \\
f & 0 & 0
\end{array}\right), \quad \phi_{-1}=\left(\begin{array}{cccc}
f e & 0 & 0 & 0 \\
a f & e & f & 0 \\
0 & 0 & e & f
\end{array}\right) .
$$

Hence $\phi_{-1}={ }^{t} \phi_{1}$ (transposed in the sense appropriate to the exterior algebra), and $\phi_{-2}={ }^{t} \phi_{2}$ as well.

Finally, by Theorem $0.1, R \pi_{*} \mathcal{F}=\left(A \otimes_{E} \mathbf{T}(\mathcal{F})\right)_{0}$ is the complex

$$
0 \longrightarrow A^{1} \stackrel{(a)}{\longrightarrow} A^{1} \longrightarrow 0 .
$$

Similar computations can be done very quickly for much larger examples by Macaulay2.

Example 5.2 (Vector Bundles on $\mathbb{P}^{1}$ ). More generally, consider the family of globally generated vector bundles of rank $r$ and degree $d$ on $\mathbb{P}^{1}$. The most special bundle in this family is $\mathcal{F}_{0}=\mathcal{O}^{r-1} \oplus \mathcal{O}(d)$. Every other bundle in this family arises as an extension

$$
0 \rightarrow \mathcal{O}^{r-1} \rightarrow \mathcal{F}_{a} \rightarrow \mathcal{O}(d) \rightarrow 0
$$

with $a \in \operatorname{Ext}^{1}\left(\mathcal{O}(d), \mathcal{O}^{r}\right) \cong \mathrm{H}^{1}\left(\mathcal{O}^{r-1}(-d)\right) \cong \mathrm{H}^{0}\left(\mathcal{O}(d-2)^{r-1}\right)^{*}$. Thus as a base space of this family we may choose $\operatorname{Spec} A$ with

$$
A=K\left[a_{i}^{s}, 0 \leq i \leq d-2,1 \leq s \leq r-1\right],
$$

and then take $\mathcal{F}$ to be the universal extension on $\mathbb{P}^{1} \times A$.

We will specify $\mathcal{F}$ explicitly via its Beilinson monad. The Tate resolution has Betti diagram

\begin{tabular}{r|cccccccc}
$i-j \backslash i$ & $-d-1$ & $-d$ & $-d+1$ & $\ldots$ & -2 & -1 & 0 & 1 \\
\hline 1 & $d(r-1)+r$ & $d(r-1)$ & $(d-1)(r-1)$ & $\ldots$ & $2(r-1)$ & $r-1$ & & \\
0 & & 1 & 2 & $\ldots$ & $d-1$ & $d$ & $d+r$ & $d+2 r$
\end{tabular}

at the special point $0 \in \operatorname{Ext}^{1}\left(\mathcal{O}(d), \mathcal{O}^{r-1}\right)$. A few examples computed with Macaulay2 make it possible to guess the pattern of the differentials, which we now are going to verify. The differentials of

$$
\mathbf{T}(\mathcal{O}): \ldots \stackrel{{ }^{t} C^{3}}{\longrightarrow} E^{2}(3) \stackrel{{ }^{t} C^{2}}{\longrightarrow} E(2) \stackrel{(e f)}{\longrightarrow} E \stackrel{C^{1}}{\longrightarrow} E^{2}(-1) \stackrel{C^{2}}{\longrightarrow} E^{3}(-2) \stackrel{C^{3}}{\longrightarrow} \cdots
$$

are given by special $(\ell+1) \times \ell$ Toeplitz (or Hankel) matrices

$$
C^{\ell}=\left(\begin{array}{ccccc}
e & 0 & & \ldots & 0 \\
f & e & \ddots & & \vdots \\
0 & f & \ddots & \ddots & \\
\vdots & \ddots & \ddots & e & 0 \\
& & \ddots & f & e \\
0 & \ldots & & 0 & f
\end{array}\right)
$$


and their transpositions. Hence $\mathbf{T}(\mathcal{F})$ is a deformation of the complex $\mathbf{T}\left(\mathcal{F}_{0}\right)=$ $\bigoplus_{1}^{r-1} \mathbf{T}(\mathcal{O}) \oplus \mathbf{T}(\mathcal{O})[d](d)$ built from Toeplitz matrices. To describe the most relevant piece we consider for pairs $(k, \ell)$ with $k+\ell=d$ the $k \times \ell$ Hankel matrices

$$
B_{k \ell}^{s}:=\left(\begin{array}{ccccc}
a_{0}^{s} & a_{1}^{s} & a_{2}^{s} & \ldots & a_{\ell-1}^{s} \\
a_{1}^{s} & a_{2}^{s} & a_{3}^{s} & \ldots & a_{\ell}^{s} \\
\vdots & \vdots & \vdots & \ddots & \vdots \\
a_{k-1}^{s} & a_{k}^{s} & a_{k+1}^{s} & \ldots & a_{k+\ell-2}^{s}
\end{array}\right) .
$$

Proposition 5.3. The $-(k+1)$-th differential

$$
E(k+2)^{(k+1)(r-1)} \oplus E(k+1)^{\ell} \stackrel{D}{\longrightarrow} E(k+1)^{k(r-1)} \oplus E(k)^{\ell+1}
$$

in the Tate resolution of $\mathcal{F}$ for $1 \leq k \leq d-1$ and $\ell=d-k$ is given by the block matrix

$$
D=D^{-k-1}=\left(\begin{array}{cccc|c}
{ }^{t} C^{k} & 0 & \ldots & 0 & B_{k \ell}^{1} \\
0 & { }^{t} C^{k} & \ddots & \vdots & B_{k \ell}^{2} \\
\vdots & \ddots & \ddots & 0 & \vdots \\
0 & \ldots & 0 & { }^{t} C^{k} & B_{k \ell}^{r-1} \\
\hline 0 & \ldots & 0 & 0 & -C^{\ell}
\end{array}\right)
$$

(in suitable coordinates).

Proof. We first prove that the matrices $D^{-d}, D^{-d+1}, \ldots, D^{-2}$ define a complex $\mathbf{T}$. Indeed $D^{-k} \cdot D^{-k-1}=0$ holds because

$$
{ }^{t} C^{k-1} \cdot B_{k \ell}^{s}-B_{k-1, \ell+1}^{s} \cdot C^{\ell}=0 .
$$

With the relative Beilinson monads we can recover the corresponding coherent sheaf from any two consecutive matrices: The monad $\mathbf{U}(\mathbf{T}(-k)[-k])$ is the total complex of a double complex

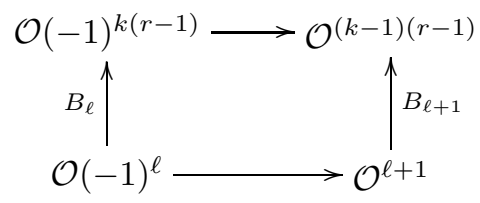

whose rows do not depend on the parameters. Here

$$
B_{\ell}=\left(\begin{array}{c}
B_{k \ell}^{1} \\
\vdots \\
B_{k \ell}^{r-1}
\end{array}\right) .
$$

The homology of the top row, which is a subcomplex, is

$$
\mathcal{O}(-k)^{r-1}=\operatorname{ker}\left(\mathcal{O}(-1)^{k(r-1)} \rightarrow \mathcal{O}^{(k-1)(r-1)}\right),
$$

while the coorresponding quotient complex, the bottom row, has homology

$$
\operatorname{coker}\left(\mathcal{O}(-1)^{\ell} \rightarrow \mathcal{O}^{\ell+1}\right)=\mathcal{O}(\ell)=\mathcal{O}(d-k) .
$$

Thus $\mathrm{H}^{*} \mathbf{U}(\mathbf{T}(-k)[-k])=\mathrm{H}^{0} \mathbf{U}(\mathbf{T}(-k)[-k])=: \mathcal{F}_{k}$ and the homology of the monad $\mathbf{U}(\mathbf{T}(-k)[-k])$ fits into a short exact sequence

$$
0 \rightarrow \mathcal{O}(-k)^{r-1} \longrightarrow \mathcal{F}_{k} \longrightarrow \mathcal{O}(d-k) \rightarrow 0
$$


Since the complex defined by the $D^{-d}, \ldots, D^{-2}$ has the right Betti number and the matrices have linearly independent rows, we conclude that they form part of the Tate resolution of a single sheaf $\mathcal{F}$, and, that $\mathcal{F}_{k}=\mathcal{F}(-k)$ with the extensions $\left(*_{k+1}\right)=\left(*_{k}\right) \otimes \mathcal{O}(-1)$. To prove that $\mathcal{F}$ is the universal extension, it suffices to prove this for any one of the sheaves $\mathcal{F}_{k}=\mathcal{F}(-k)$. We choose $\mathcal{F}_{d}=\operatorname{ker}\left(\mathcal{O}(-1)^{d(r-1)} \oplus \mathcal{O} \stackrel{D^{-d}}{\longrightarrow} \mathcal{O}^{(d-1)(r-1)}\right)$. The boundary map in

$$
\rightarrow \mathrm{H}^{0}\left(\mathbb{P}_{A}^{1}, \mathcal{F}_{d}\right) \rightarrow \mathrm{H}^{0}\left(\mathbb{P}_{A}^{1}, \mathcal{O}\right) \rightarrow \mathrm{H}^{1}\left(\mathbb{P}_{A}^{1}, \mathcal{O}(-d)^{r-1}\right) \rightarrow
$$

is the composition

$$
\mathrm{H}^{0}\left(\mathbb{P}_{A}^{1}, \mathcal{O}\right) \stackrel{B_{1}}{\longrightarrow} \mathrm{H}^{0}\left(\mathbb{P}_{A}^{1}, \mathcal{O}^{(d-1)(r-1)}\right) \cong \mathrm{H}^{1}\left(\mathbb{P}_{A}^{1}, \mathcal{O}(-d)\right)
$$

So the boundary map vanishes at a point $a$ iff all coordinates $a_{i}^{s}$ vanish at $a$. We conclude that the $a_{i}^{s}$ represent linearly independent extension classes, and, since we have the right number $(d-1)(r-1)$ of parameters, that $\left(*_{d}\right)$ is the universal extension.

Corollary 5.4. Let $\mathcal{F}$ on $\mathbb{P}_{A}^{1}$ be the universal extension

$$
0 \rightarrow \mathcal{O}^{r-1} \rightarrow \mathcal{F} \rightarrow \mathcal{O}(d) \rightarrow 0
$$

Then for each $k$ in the range $1 \leq k \leq d-1$ the direct image complex of $\mathcal{F}(-k-1)$ is

$$
R \pi_{*} \mathcal{F}(-k-1): 0 \rightarrow A^{d-k} \stackrel{B_{d-k}}{\longrightarrow} A^{k(r-1)} \rightarrow 0 \text { with } B_{d-k}=\left(\begin{array}{c}
B_{k, d-k}^{1} \\
\vdots \\
B_{k, d-k}^{r-1}
\end{array}\right)
$$

Outside this range the direct image complexes are concentrated in one degree.

Example 5.5 (Strata in the case $(d, r)=(6,3))$. The corollary allows us to describe the loci of extension classes of a given splitting type in $\operatorname{Ext}^{1}\left(\mathcal{O}(d), \mathcal{O}^{r-1}\right)$ by rank conditions on the matrices $B_{k}$ in the various direct image complexes.

We treat the example $(d, r)=(6,3)$. The possible splitting type corresponds to a partition of $d$ into at most $r$ parts. In our special case, these are the following strata with an arrow $p \rightarrow q$ indicating that the strata $p$ lies in the closure of the strata $q$ :

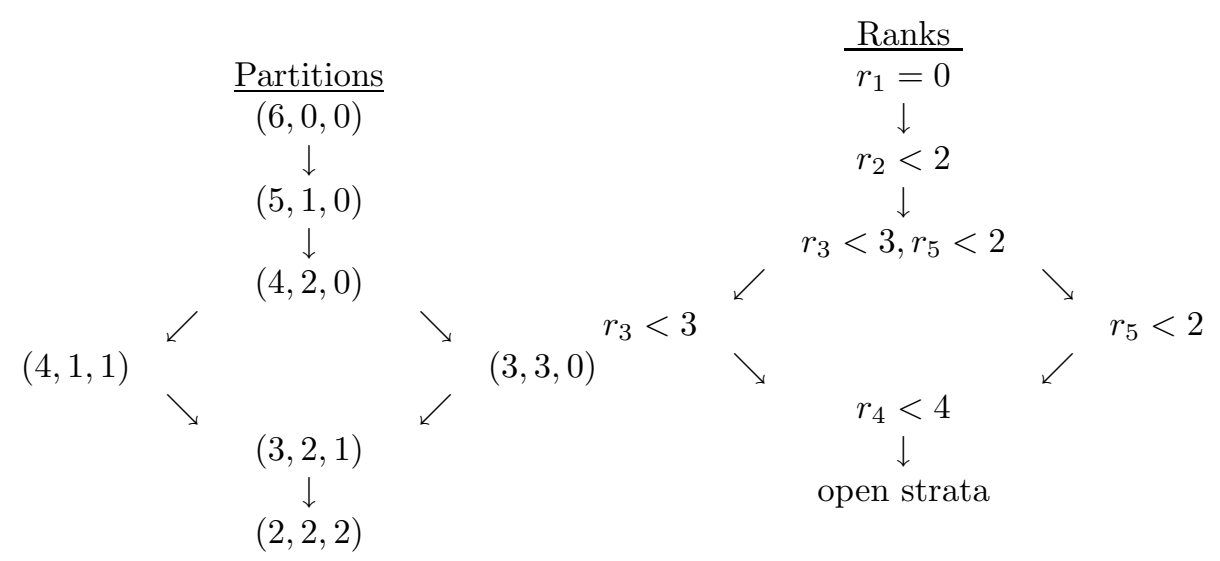


In which strata an extension $a$ lies is determined by the ranks $r_{i}=\operatorname{rank} B_{i}(a)$ of the $(d-i)(r-1) \times i$ matrices $B_{i}$ evaluated at $a$.

\begin{tabular}{r|cccccccc}
$i-j \backslash i$ & -7 & -6 & -5 & -4 & -3 & -2 & -1 & 0 \\
\hline 1 & 15 & 12 & 10 & 8 & 6 & 4 & 2 & \\
0 & & 1 & 2 & 3 & 4 & 5 & 6 & 9
\end{tabular}

Indeed by the Base Change Theorem 4.4 and its Corollary 4.5 the $r_{i}$ determine the dimensions $h^{0}\left(\mathbb{P}^{1}, \mathcal{F}_{a}(-i-1)\right)$, which in turn determine the splitting type according to the following elementary lemma.

Lemma 5.6. Let $\mathcal{E}$ be a vector bundle on $\mathbb{P}^{1}$, and let

$$
h=h_{\mathcal{E}}: \mathbb{Z} \rightarrow \mathbb{Z}, n \mapsto h^{0} \mathcal{E}(n)
$$

be its Hilbert function. Then

$$
\mathcal{E} \cong \bigoplus_{j \in \mathbb{Z}} \mathcal{O}(-j)^{h^{\prime \prime}(j)}
$$

where $h^{\prime \prime}(j)=h(j)-2 h(j-1)+h(j-2)$ denotes the second difference function.

The claim on the strata indicated in the table above follows. Note that there is no single matrix whose rank determines all splitting types, and for one strata there is not a single matrix on which a rank condition gives the defining equations.

To exhibit the beautiful pattern in this family of matrices more visibly, we drop the upper index notation $a_{i}^{s}$ and use coordinates $a_{0}, \ldots, a_{4}, b_{0}, \ldots, b_{4}$ instead. With this notation we have

$$
B_{5}=\left(\begin{array}{lllll}
a_{0} & a_{1} & a_{2} & a_{3} & a_{4} \\
b_{0} & b_{1} & b_{2} & b_{3} & b_{4}
\end{array}\right), B_{4}=\left(\begin{array}{cccc}
a_{0} & a_{1} & a_{2} & a_{3} \\
a_{1} & a_{2} & a_{3} & a_{4} \\
\hline b_{0} & b_{1} & b_{2} & b_{3} \\
b_{1} & b_{2} & b_{3} & b_{4}
\end{array}\right), B_{3}=\left(\begin{array}{lll}
a_{0} & a_{1} & a_{2} \\
a_{1} & a_{2} & a_{3} \\
a_{2} & a_{3} & a_{4} \\
\hline b_{0} & b_{1} & b_{2} \\
b_{1} & b_{2} & b_{3} \\
b_{2} & b_{3} & b_{4}
\end{array}\right) .
$$

There are various relations between the ideals of minors of these matrices. The most interesting one is the primary decomposition of ideal $3 \times 3$ minors of the square matrix $B_{4}$ :

$$
\operatorname{minors}\left(3, B_{4}\right)=\operatorname{minors}\left(3, B_{3}\right) \cap \operatorname{minor} s\left(2, B_{5}\right) \text {. }
$$

We discovered this relation by computation using Macaulay2, which provides a proof in a few positive characteristics; the relation was recently proven noncomputationally by Moty Katzman Ka05, Section 3]. 
In terms of projective geometry the closed strata have the following descriptions as cones over projective varieties:

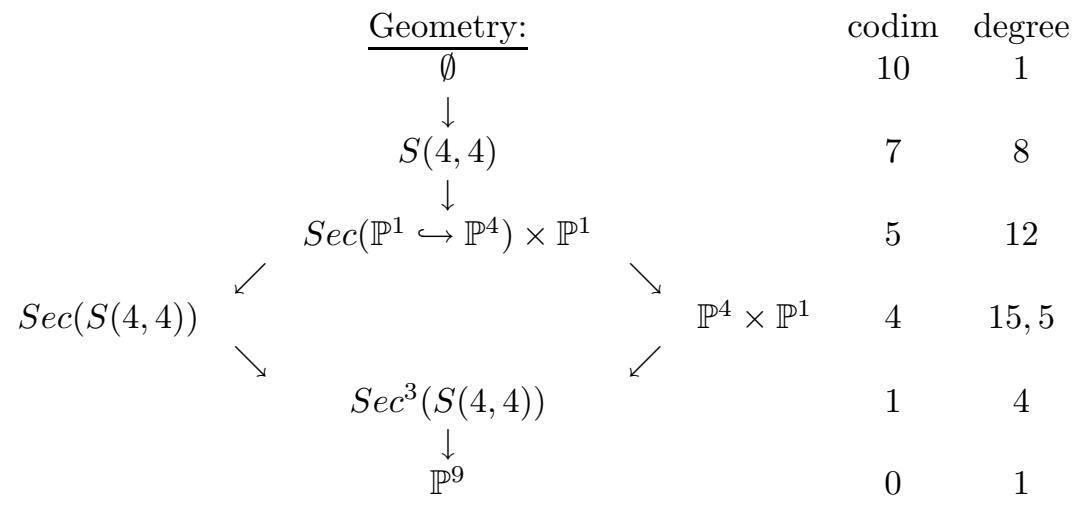

Here $S(4,4) \subset \mathbb{P}^{4} \times \mathbb{P}^{1} \subset \mathbb{P}^{9}$ denotes the 2-dimensional rational normal scroll defined by the $2 \times 2$ minors of the matrix

$$
{ }^{t} B_{2}=\left(\begin{array}{cccc|cccc}
a_{0} & a_{1} & a_{2} & a_{3} & b_{0} & b_{1} & b_{2} & b_{3} \\
a_{1} & a_{2} & a_{3} & a_{4} & b_{1} & b_{2} & b_{3} & b_{4}
\end{array}\right)
$$

and $\operatorname{Sec}(X)$, respectively $\operatorname{Sec}^{3}(X)$, refers to the secant, respectively 3-secant, variety of $X$. Note that the fibers of $S(4,4) \rightarrow \mathbb{P}^{1}$ are rational normal curves of degree 4 , and that

$$
S(4,4) \cong \mathbb{P}^{1} \times \mathbb{P}^{1} \stackrel{|(4,1)|}{\hookrightarrow} \mathbb{P}^{9} .
$$

We return to the general case of the versal deformation of $\mathcal{O}^{r-1} \oplus \mathcal{O}(d)$. The analysis above shows in general that, in the deformations of $O(d) \oplus \mathcal{O}^{r-1}$, the stratum of bundles isomorphic to $\mathcal{O}(d-1) \oplus \mathcal{O}(1) \oplus \mathcal{O}^{r-2}$ is isomorphic to the rational normal scroll

$$
S(d-2, \ldots, d-2) \cong\left(\mathbb{P}^{1} \hookrightarrow \mathbb{P}^{d-2}\right) \times \mathbb{P}^{r-2} \subset \mathbb{P}^{d r-d-r} .
$$

We would like to have a geometric description of the strata in general, for example in terms of secant constructions such as

$$
\operatorname{Sec}^{b}\left(\operatorname{Sec}^{a}\left(\mathbb{P}^{1} \hookrightarrow \mathbb{P}^{d-2}\right) \times \mathbb{P}^{r-2}\right) .
$$

Though we don't have such a geometric description, we can at least give an algebraic one. If a closed point $p \in \operatorname{Spec} A$ corresponds to a bundle with a certain splitting type, then from the cohomology of the bundle we see that the matrices in the pushforward of the $R \pi_{*} \mathcal{F}(\ell)$, for various $\ell$, satisfy some rank conditions at $p$. We conjecture that the determinantal equations derived from these rank conditions actually generate the radical ideal of closure of the stratum of vector bundles that split in this way. The following stronger statement includes the case of certain unions of strata:

Conjecture 5.1. Let $B_{1}, \ldots, B_{d-1}$ be the nontrivial matrices of the direct image complexes of the versal deformation of $\mathcal{O}^{r-1} \oplus \mathcal{O}(d)$. For any collection of positive 
integers $r_{k_{1}}, \ldots, r_{k_{s}}$, the ideal

$$
\sum_{t=1}^{s} \operatorname{minors}\left(r_{k_{t}}, B_{k_{t}}\right)
$$

is radical.

The case $r=2$ was asserted by Room Roo38 and proven in characteristic 0 by Peskine and Szpiro. See Conca Con98 for a general proof and other references.

The minimal primes of the ideal

$$
\sum_{k=1}^{d-1} \operatorname{minors}\left(r_{k}, B_{k}\right)
$$

are easy to describe and are (radicals of) ideals of the same form. First of all, the locus of extensions

$$
0 \rightarrow \mathcal{O}^{r-1} \rightarrow \mathcal{E} \rightarrow \mathcal{O}(d) \rightarrow 0
$$

on $\mathbb{P}_{K}^{1}$ such that $\mathcal{E}$ has a given splitting type, $\mathcal{E} \cong \bigoplus \mathcal{O}\left(a_{i}\right)$, is always irreducible. Its closure is the locus of extensions such that each twist of $\mathcal{E}$ has at least as many global sections as the corresponding twist of $\bigoplus \mathcal{O}\left(a_{i}\right)$. The corresponding prime ideal is thus the radical of the corresponding sum of ideals minors $\left(r_{k}, B_{k}\right)$. Conversely, any sum of the ideals minors $\left(r_{k}, B_{k}\right)$ defines the locus of extensions such that various twists of $\mathcal{E}$ have at least a certain number of independent global sections. With some care one can give the irredundant decompositions in terms of splitting types.

In particular, the prime ideals of the form

$$
\operatorname{rad}\left(\sum_{k=1}^{d-1} \operatorname{minors}\left(r_{k}, B_{k}\right)\right)
$$

are precisely the primes that define the closures of the strata of points $p \in \operatorname{Spec} A$ where $\mathcal{F}_{p}$ has a given splitting type. If the conjecture is true, of course, these sums of determinantal ideals are already radical.

One can see from this analysis that an ideal minors $\left(r_{k_{t}}, B_{k_{t}}\right)$ can have components of different dimensions; in particular, it need not be Cohen-Macaulay.

It is worth remarking that one can also treat this family of examples without exterior methods. For example, from the exact sequence $0 \rightarrow \mathcal{O}^{r-1}(-k) \rightarrow \mathcal{F}(-k) \rightarrow$ $\mathcal{O}(d-k) \rightarrow 0$ we get a triangle of direct images that expresses $R \pi_{*} \mathcal{F}(-k)$ as the mapping cone of a certain map

$$
R \pi_{*} \mathcal{O}(d-k) \rightarrow R \pi_{*} \mathcal{O}^{r-1}(-k)[1] .
$$

At most one of the modules $R^{i} \pi_{*} \mathcal{O}(d-k)=\mathrm{H}^{i}(\mathcal{O}(d-k))$ is nonzero, and similarly for $\mathcal{O}^{r-1}(-k)$, so each of $R \pi_{*} \mathcal{O}(d-k)$ and $R \pi_{*} \mathcal{O}^{r-1}(-k)[1]$ reduces to a single free module. In the "interesting" range $-d \leq k \leq-2$ where both these modules are nonzero, the map between the modules is the connecting homomorphism $\mathrm{H}^{0}(\mathcal{O}(d-k)) \rightarrow \mathrm{H}^{1}\left(\mathcal{O}^{r-1}(-k)\right)$ that we have called $B_{d-k+1}$. This connecting homomorphism is easy to compute concretely, especially since the computation reduces to the case $r=1$.

Example 5.7 (Blowup of an elliptic singularity). For an example that seems much harder to treat by simple methods, consider the singularity defined by

$$
B=\left\{a b c+a^{4}+b^{4}+c^{4}=0\right\} \subset \mathbb{A}^{3} .
$$


The singularities of $B$ are resolved by blowing up the origin $\sigma: \widetilde{\mathbb{A}}^{3} \subset \mathbb{P}^{2} \times \mathbb{A}^{3} \rightarrow \mathbb{A}^{3}$ once. We consider the strict $\bar{B}=\overline{\sigma^{-1}(B \backslash\{0\})} \subset \mathbb{P}^{2} \times B$ and the total transform $B^{\prime}=\sigma^{-1}(B)$ of $B$. The Tate resolution of $\mathcal{O}_{\bar{B}}$ has the Betti diagram

\begin{tabular}{|c|c|c|c|c|c|c|c|c|c|}
\hline$i-j \backslash i$ & -4 & -3 & -2 & -1 & 0 & 1 & 2 & 3 & 4 \\
\hline 1 & 15 & 12 & 9 & 6 & 3 & 1 & & & \\
\hline 0 & 27 & 21 & 15 & 9 & 4 & 3 & 6 & 9 & 12 \\
\hline-1 & 21 & 15 & 9 & 4 & 4 & 9 & 15 & 21 & $*$ \\
\hline-2 & 15 & 9 & 4 & 4 & 9 & 15 & 21 & $*$ & \\
\hline &.$^{\circ}$ & 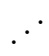 &.$^{\circ}$ & . & $\therefore$ &.$^{\circ}$ &.$^{\circ}$ &.$^{\circ}$ & \\
\hline
\end{tabular}

with eventually periodic diagonals by Eisenbud [Eis80].

The Tate resolution of $\mathcal{O}_{B^{\prime}}$ looks quite different:

\begin{tabular}{r|ccccccccc}
$i-j \backslash i$ & -4 & -3 & -2 & -1 & 0 & 1 & 2 & 3 & 4 \\
\hline 2 & 10 & 6 & 3 & 1 & & & & & \\
1 & 15 & 8 & 3 & & & & & & \\
0 & 6 & 3 & 1 & 1 & 1 & 3 & 6 & 10 & 15 \\
-1 & & & & & 3 & 8 & 15 & 24 & $*$ \\
-2 & & & & 1 & 3 & 6 & 10 & $*$ & $*$
\end{tabular}

with bounded $\mathbf{T}^{k}$, although $A=\mathcal{O}_{B, 0}$ does not have finite projective dimension. A closer inspection of the complexes gives

$$
R \pi_{*} \mathcal{O}_{B^{\prime}}(k)=R \sigma_{*} \mathcal{O}_{\widetilde{\mathbb{A}}^{3}}(k) \otimes_{\mathcal{O}_{\mathbb{A}^{3}}} \mathcal{O}_{B}
$$

a formula which holds, although the Base Change Theorem 4.4 does not apply. So for $k \geq 0$ we have

$$
R^{0} \pi_{*} \mathcal{O}_{B^{\prime}}(k)=\mathfrak{m}_{\mathbb{A}^{3}, 0}^{k} \otimes_{\mathcal{O}_{\mathbb{A}^{3}, 0}} \mathcal{O}_{B},
$$

while

$$
R^{0} \pi_{*} \mathcal{O}_{\bar{B}}(k)=\mathfrak{m}_{B, 0}^{k} .
$$

Example 5.8 (Variety of Complexes). One might hope that the direct image of a vector bundle on $\mathbb{P}_{A}^{n}$, say in the case of a local ring $A$, would have special properties compared to an arbitrary complex of free $A$-modules. But it turns out that such images are general; in fact, one can get any complex as the push-forward of quite a simple bundle:

Theorem 5.9. Every bounded minimal free complex

$$
0 \rightarrow A^{\beta_{0}} \rightarrow A^{\beta_{1}} \rightarrow \ldots \rightarrow A^{\beta_{n}} \rightarrow 0
$$

over a local Noetherian ring arises as the direct image complex of a locally free sheaf on $\mathbb{P}_{A}^{n}$.

By flat base change, it suffices to prove the result for the generic complex

$$
\mathbb{F}: \quad 0 \rightarrow B^{\beta_{0}} \rightarrow \cdots \rightarrow B^{\beta_{n}} \rightarrow 0
$$

defined over the ring

$$
B=\mathbb{Z}\left[a_{i j}^{p} ; 1 \leq i \leq \beta_{p+1}, 1 \leq j \leq \beta_{p}, p=0, \ldots, n-1\right] /\left(\left(a_{i j}^{p+1}\right)\left(a_{j k}^{p}\right)=0\right),
$$

with the map $B^{\beta_{p}} \rightarrow B^{\beta_{p+1}}$ given by the map with matrix $\left(a_{j k}^{p}\right)$. The $\operatorname{ring} B$ is the affine coordinate ring of the "variety of complexes" (see for example DS81]). The next theorem is thus a strengthening of Theorem 5.9. 
Theorem 5.10. The generic complex $\mathbb{F}$ over the ring $B$ above is the direct image of the versal deformation of the vector bundle

$$
\bigoplus_{p=0}^{n}\left(\wedge^{p} \Omega_{\mathbb{P}_{\mathbb{Z}}^{n} / \mathbb{Z}}\right)^{\beta_{p}}
$$

Note that Example 5.1 is a special case of Theorem 5.10, a complex of length 1 with free modules of rank 1 ,

$$
0 \rightarrow B^{1} \rightarrow B^{1} \rightarrow 0
$$

Proof. To simplify the notation, we write $\Omega^{i}$ for $\wedge^{i} \Omega_{\mathbb{P}_{\mathbb{Z}}^{n} / \mathbb{Z}}$. We first show that the ring $B$ (or more properly its completion at the origin) is the base of the versal deformation $\mathcal{F}$ of the vector bundle $\mathcal{F}_{0}=\bigoplus_{p=0}^{n}\left(\Omega^{p}\right)^{\beta_{p}}$.

Proposition 5.11.

$$
\begin{aligned}
& \operatorname{Ext}^{1}\left(\Omega^{p}, \Omega^{q}\right)=\left\{\begin{array}{lc}
\mathrm{H}^{1}\left(\Omega^{1}\right)=\mathbb{Z} & \text { if } 1 \leq q=p+1 \leq n, \\
0 & \text { otherwise } ;
\end{array}\right. \\
& \operatorname{Ext}^{2}\left(\Omega^{p}, \Omega^{q}\right)=\left\{\begin{array}{lc}
\mathrm{H}^{2}\left(\Omega^{2}\right)=\mathbb{Z} & \text { if } 2 \leq q=p+2 \leq n, \\
0 & \text { otherwise. }
\end{array}\right.
\end{aligned}
$$

Thus the tangent space of the versal deformation of $\mathcal{F}_{0}$ is

$$
\operatorname{Ext}^{1}\left(\mathcal{F}_{0}, \mathcal{F}_{0}\right)=\bigoplus_{p=0}^{n-1} \operatorname{Hom}\left(\mathbb{Z}^{\beta_{p}}, \mathbb{Z}^{\beta_{p+1}}\right),
$$

and the obstruction space is

$$
\operatorname{Ext}^{2}\left(\mathcal{F}_{0}, \mathcal{F}_{0}\right)=\bigoplus_{p=0}^{n-2} \operatorname{Hom}\left(\mathbb{Z}^{\beta_{p}}, \mathbb{Z}^{\beta_{p+2}}\right) .
$$

The obstruction map is the one given by composition

$$
\left(\phi_{0}, \ldots, \phi_{n-1}\right) \mapsto\left(\phi_{1} \cdot \phi_{0}, \ldots, \phi_{n-1} \cdot \phi_{n-2}\right),
$$

and the base space $B$ of the deformation is the coordinate ring of the variety of complexes of free modules of ranks $\beta_{0}, \ldots, \beta_{n}$.

Proof. The generator of $\operatorname{Ext}^{1}\left(\Omega^{p-1}, \Omega^{p}\right)$ corresponds to the extension

$$
0 \rightarrow \Omega^{p} \rightarrow \bigwedge^{p}\left(\mathcal{O}^{n+1}(-1)\right) \rightarrow \Omega^{p-1} \rightarrow 0
$$

that appears in the Koszul complex, and the generator of $\operatorname{Ext}^{2}\left(\Omega^{p-1}, \Omega^{p+1}\right)$ can also be realized in the Koszul complex as the extension

$$
0 \rightarrow \Omega^{p+1} \rightarrow \bigwedge^{p+1}\left(\mathcal{O}^{n+1}(-1)\right) \rightarrow \bigwedge^{p}\left(\mathcal{O}^{n+1}(-1)\right) \rightarrow \Omega^{p-1} \rightarrow 0
$$

which is thus the Yoneda product of the generators of $\operatorname{Ext}^{1}\left(\Omega^{p-1}, \Omega^{p}\right)$ and $\operatorname{Ext}^{1}\left(\Omega^{p}, \Omega^{p+1}\right)$.

The quadratic obstruction map

$$
\operatorname{Ext}^{1}\left(\mathcal{F}_{0}, \mathcal{F}_{0}\right) \rightarrow \operatorname{Ext}^{2}\left(\mathcal{F}_{0}, \mathcal{F}_{0}\right)
$$


is the map given by squaring, using the Yoneda product. The only nonzero contributions come from the maps

$$
\begin{gathered}
\operatorname{Ext}^{1}\left(\left(\Omega^{p-1}\right)^{\beta_{p-1}},\left(\Omega^{p}\right)^{\beta_{p}}\right) \times \operatorname{Ext}^{1}\left(\left(\Omega^{p}\right)^{\beta_{p}},\left(\Omega^{p+1}\right)^{\beta_{p+1}}\right) \\
\downarrow \\
\operatorname{Ext}^{2}\left(\left(\Omega^{p-1}\right)^{\beta_{p-1}},\left(\Omega^{p+1}\right)^{\beta_{p+1}}\right),
\end{gathered}
$$

which, with a natural choice of bases, is matrix multiplication by the computation above, so we see that the obstructions to second-order deformations are as claimed.

To see that there are no higher terms in the equations of the base space of the versal deformation, we observe that $\mathcal{F}_{0}$ can be graded by giving a summand $\Omega^{p}$ of degree $p$. With this grading, each nonzero element of $\operatorname{Ext}^{1}\left(\mathcal{F}_{0}, \mathcal{F}_{0}\right)$ is of degree 1 and the elements of $\operatorname{Ext}^{2}\left(\mathcal{F}_{0}, \mathcal{F}_{0}\right)$ are similarly of degree 2 . Since the versal deformation must be homogeneous for this grading, no higher-degree terms can occur in the equations.

We also make use of the computation of the Tate resolution associated with the sheaf $\Omega^{p}$ : from [EFS03, Proposition 5.5], we know it has the form

\begin{tabular}{r|lcccccc}
$i-j \backslash i$ & $\ldots$ & $p-2$ & $p-1$ & $p$ & $p+1$ & $p+2$ & $\ldots$ \\
\hline$n$ & $\ldots$ & $u_{p-2}$ & $u_{p-1}$ & 0 & 0 & 0 & $\ldots$ \\
$\vdots$ & & & & & & & \\
$p+2$ & & & & 0 & & & \\
$p+1$ & & & & 0 & & & \\
$p$ & & & & 1 & & & \\
$p-1$ & & & & 0 & & & \\
$p-2$ & & & & 0 & & & \\
$\vdots$ & & & & & & & \\
0 & $\ldots$ & 0 & 0 & 0 & $v_{p+1}$ & $v_{p+2}$ & $\ldots$
\end{tabular}

with $u_{p-1}=\left(\begin{array}{c}n+1 \\ n+1-p\end{array}\right)$ and $v_{p+1}=\left(\begin{array}{c}n+1 \\ p+1\end{array}\right)$.

It remains to show that the generic complex over $B$ is $R \pi_{*} \mathcal{F}$, where $\mathcal{F}$ on $\mathbb{P}_{A}^{n}$ is the versal deformation of $\mathcal{F}_{0}=\bigoplus_{p=0}^{n} \bigoplus_{j=1}^{\beta_{p}} \Omega^{p}$ on $\mathbb{P}_{\mathbb{Z}}^{n}$.

From the Betti diagram above we see that the Tate resolution of $\mathcal{F}_{0}$ has a Betti diagram of the following shape, where the entries not shown in the center of the table are all zero:

\begin{tabular}{r|rrrrrrrrr}
$i-j \backslash i$ & -1 & 0 & 1 & 2 & $\ldots$ & $n-1$ & $n$ & $n+1$ & $\ldots$ \\
\hline$n$ & $\delta_{-1}$ & $\delta_{0}$ & $\delta_{1}$ & $\delta_{2}$ & $\ldots$ & $\delta_{n-1}$ & $\beta_{n}$ & & \\
$n-1$ & & & & & & $\beta_{n-1}$ & & & \\
$\vdots$ & & & & &.. & & & & \\
2 & & & & $\beta_{2}$ & & & & & \\
1 & & & $\beta_{1}$ & & & & & & \\
0 & & $\beta_{0}$ & $\gamma_{1}$ & $\gamma_{2}$ & $\ldots$ & $\gamma_{n-1}$ & $\gamma_{n}$ & $\gamma_{n+1}$ & $\ldots$
\end{tabular}

Because the base ring $B$ is naturally graded, we can use the result for local rings in Corollary 4.5 to conclude that the Tate resolution of $\mathcal{F}$ has the same entries. We want to prove that the component

$$
E^{\beta_{p}} \rightarrow E^{\beta_{p+1}}
$$


of the differential $\mathbf{T}(\mathcal{F})^{p} \rightarrow \mathbf{T}(\mathcal{F})^{p+1}$, which appears on the diagonal, is given by the matrix $\left(a_{i j}^{p}\right)$.

Consider the subspace corresponding to the factor ring $B_{p}=B / I_{p}$ with ideal $I_{p}$ generated by the linear forms $\left(a_{i j}^{q} ; q \neq p\right)$. The restriction of the family $\mathcal{F}$ to this subspace is

$$
\mathcal{F} \otimes \mathcal{O}_{B_{p}} \cong\left(\bigoplus_{q \neq p, p+1}\left(\Omega^{q}\right)^{\beta_{q}}\right) \oplus \mathcal{G}
$$

where $\mathcal{G}$ is the versal deformation of

$$
\mathcal{G}_{0}=\left(\Omega^{p}\right)^{\beta_{p}} \oplus\left(\Omega^{p+1}\right)^{\beta_{p+1}} .
$$

The Tate resolution of $\mathcal{G}$ is a deformation of the Tate resolution of $\mathcal{G}_{0}$; the shape of its Tate resolution can be deduced from that of $\Omega^{p}$ and that of $\Omega^{p+1}$ as above.

We focus on the two differentials

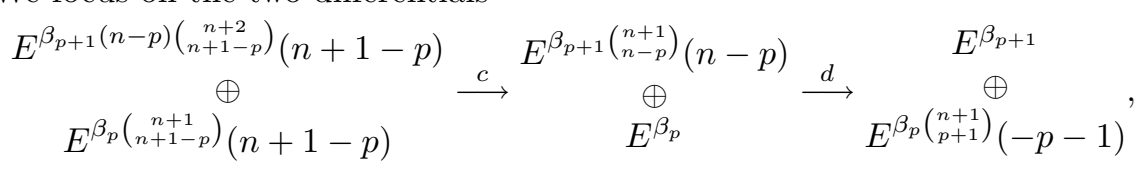

with

$$
c=\left(\begin{array}{cc}
c_{1} & c_{12} \\
0 & c_{2}
\end{array}\right) \text { and } d=\left(\begin{array}{cc}
d_{1} & d_{12} \\
0 & d_{2}
\end{array}\right) .
$$

By [EFS03, Proposition 5.5] we know that $c_{2}$ is a direct sum of $\beta_{p}$ copies of the $1 \times\left(\begin{array}{c}n+1 \\ n+1-p\end{array}\right)$ matrix consisting of all monomials of degree $n+1-p$ in the exterior variables $e_{0}, \ldots, e_{n}$, and that $d_{2}$ consists of a direct sum of $\beta_{p}$ copies of the $\left(\begin{array}{c}n+1 \\ p+1\end{array}\right) \times 1$ matrix consisting of all monomials of degree $p+1$ in the exterior variables. (So $d_{2} \cdot c_{2}=0$ because the composition has degree $n+2$.) Similarly $d_{1}$ is a direct sum of $\beta_{p+1}$ copies of the $1 \times\left(\begin{array}{l}n+1 \\ n-p\end{array}\right)$ matrix of all monomials of degree $n+1-(p+1)$ in $e_{0}, \ldots, e_{n}$ and $c_{1}$ consists of $\beta_{p+1}$ copies of the linear syzygies matrix of these monomials.

The deformation sits in the components $c_{12}$ and $d_{12}$ of the matrices corresponding to the extensions. We want to prove that

$$
d_{12}=\left(a_{i j}^{p}\right)
$$

If we take this choice for $d_{12}$, then we can build a complex by taking $c_{12}$ as a suitable matrix of bihomogeneous forms in the variables $a_{i j}^{p}$ and $e_{0}, \ldots, e_{n}$, because $\left(e_{0}, \ldots, e_{n}\right)^{n+1-p} \subset\left(e_{0}, \ldots, e_{n}\right)^{n-p}$. The two differentials, deformed in this way, extend to a map of (doubly infinite) resolutions, and thus to a deformation of the whole Tate resolution. This defines a sheaf $\mathcal{G}^{\prime}$ on $\mathbb{P}^{n} \times B_{p}$.

We argue now directly that $\mathcal{G}^{\prime}$ over $B_{p}$ defined in this way is the versal deformation of $\mathcal{G}_{0}$. Indeed for any other deformation $\mathcal{G}^{\prime \prime}$ of $\mathcal{G}_{0}$ over some base space $\operatorname{Spec} T$ the direct image complex of $\mathcal{G}^{\prime \prime}$ induces a morphism $\varphi: \operatorname{Spec} T \rightarrow B_{p}$ by taking the substitution $\mathbb{Z}\left[a_{i j}^{p}\right] \rightarrow T$ obtained from the matrix $d_{12}^{\prime \prime}$ in the complex $R \pi_{*} \mathcal{G}^{\prime \prime}$ on $\operatorname{Spec} T$, and $\mathcal{G}^{\prime \prime} \cong\left(\operatorname{id}_{\mathbb{P}^{n}} \times \varphi\right)^{*} \mathcal{G}$. This proves the (semi) universal property of $\mathcal{G}^{\prime}$. It is universal because the grading of $\mathcal{G}^{\prime}$ and its base ring $\mathbb{Z}\left[a_{i j}^{p}\right]$ given by its degree in the $a_{i j}^{p}$ prevents there being any automorphisms except for conjugation of the maps $\left(a_{i j}^{p}\right)$ by invertible matrices in the obvious way. Thus $\mathcal{G}^{\prime}=\mathcal{G}$.

The Base Change Theorem 4.4 and the grading of $\mathcal{F}$ and its base ring $\mathbb{Z}\left[a_{i j}^{p} ; p=\right.$ $0, \ldots, n-1]$ by degree in the $a_{i j}^{p}$ shows that $\left(a_{i j}^{p}\right)$ occurs as a differential in $R \pi_{*} \mathcal{F}$, since $\mathcal{G}$ is a summand of $\mathcal{F} \otimes \mathcal{O}_{B_{p}}$, as required. 
Conjecture 5.2. Any bounded complex of finitely generated A-modules

$$
0 \rightarrow B_{0} \rightarrow \cdots \rightarrow B_{n} \rightarrow 0
$$

arises as the direct image complex of a family of sheaves on $\mathbb{P}_{A}^{n}$, which can be taken to be a deformation of the sheaf $\bigoplus_{p}\left(B_{p} \otimes \wedge^{p} \Omega_{\mathbb{P}_{A}^{n}} / A\right)$.

The methods above suffice to prove this for a two-term complex.

\section{ACKNOWLEDGMENT}

The authors thank Mike Stillman and Dan Grayson for their program Macaulay2 and for their quick responses to our needs. The second author thanks the MSRI for financial support and hospitality.

\section{REFERENCES}

[Bei78] Beilinson, A. A.: Coherent sheaves on $\mathbb{P}^{n}$ and problems in linear algebra. Funktsional. Anal. i Prilozhen. 12 (1978), no. 3, 68-69. MR509388 (80c:14010b)

[BGG78] Bernstein, I. N.; Gel'fand, I. M.; Gel'fand, S. I.: Algebraic vector bundles on $\mathbb{P}^{n}$ and problems of linear algebra. (Russian) Funktsional. Anal. i Prilozhen. 12 (1978), 66-67. MR.509387 (80c:14010a)

[Con98] Conca, Aldo: Straightening law and powers of determinantal ideals of Hankel matrices. Adv. Math. 138 (1998), no. 2, 263-292. MR.1645574 (99i:13020)

[DS81] De Concini, Corrado; Strickland, Elisabetta: On the variety of complexes. Adv. in Math. 41 (1981), no. 1, 57-77. MR625334 (82m:14032)

[Eis04] Eisenbud, David: The Geometry of Syzygies, Graduate Texts in Mathematics, vol. 229, 2004. MR 2103875 (2005h:13021)

[Eis80] Eisenbud, David: Homological algebra on a complete intersection, with an application to group representations. Trans. Amer. Math. Soc. 260 (1980), 35-64. MR.570778 (82d:13013)

[EFS03] Eisenbud, David: Fløystad, Gunnar; Schreyer, Frank-Olaf: Sheaf cohomology and free resolutions over exterior algebras. Trans. Amer. Math. Soc. 355 (2003), 43974426. MR 1990756 (2004f:14031)

[ES03] Eisenbud, David; Schreyer, Frank-Olaf; with an appendix by Weyman, Jerzy: Resultants and Chow forms via exterior syzygies. J. Amer. Math. Soc. 16 (2003), 537-579. MR 1969204 (2004j:14067)

[GS93] Grayson, Daniel R.; Stillman, Michael E.: Macaulay2, a software system for research in algebraic geometry, 1993-2002, available at http://www.math. uiuc. edu/Macaulay2.

[Gro63] Grothendieck, A.: Éléments de géométrie algébrique. III. Étude cohomologique des faisceaux cohérents. II. (Inst. Hautes Études Sci. Publ. Math. No. 17, 1963. 91 pp. MR 0163911 (29:1210)

[Har77] Hartshorne, Robin: Algebraic geometry. Graduate Texts in Mathematics, No. 52. Springer-Verlag, New York-Heidelberg, 1977. xvi+496 pp. MR0463157 (57:3116)

[Hoch56] Hochschild, G.: Relative homological algebra. Trans. Amer. Math. Soc. 82 (1956), 246-269. MR0080654 (18:278a)

[Ka98] Katzman, Mordechai: The complexity of Frobenius powers of ideals. J. Algebra 203 (1998), no. 1, 211-225. MR.1620654 (99e:13003)

[Ka05] Katzman, Mordechai: On ideals of minors of matrices with indeterminate entries. arXiv:math.AC/0509657v2.

[Macaulay2] Macaulay2; see Grayson and Stillman [GS93].

[Mum70] Mumford, David: Abelian varieties. Tata Institute of Fundamental Research Studies in Mathematics, No. 5; Oxford University Press, London, 1970 viii+242 pp. MR 0282985 (44:219)

[Roo38] Room, T.G.: The Geometry of Determinantal Loci, Cambridge Univ. Press, Cambridge, 1938. 
[Sm00] Smith, Gregory G.: Computing global extension modules. Symbolic computation in algebra, analysis, and geometry (Berkeley, CA, 1998). J. Symbolic Comput. 29 (2000), no. 4-5, 729-746. MR1769664 (2001h:14013)

Department of Mathematics, University of California, Berkeley, Berkeley, CaliFORNIA 94720

E-mail address: eisenbud@math.berkeley.edu

Mathematik und Informatik, Campus E 2.4, Universität des SaArlandes, D-66123 SaARBRÜCKen, Germany

E-mail address: schreyer@math.uni-sb.de 